\title{
UNIFORM ESTIMATES IN THE POINCARÉ-ARONSZAJN THEOREM ON THE SEPARATION OF SINGULARITIES OF ANALYTIC FUNCTIONS
}

\author{
V.P. HAVIN, A.H. NERSESSIAN, AND J. ORTEGA-CERDÀ
}

\begin{abstract}
We study the possibility of splitting any bounded analytic function $f$ with singularities in a closed set $E \cup F$ as a sum of two bounded analytic functions with singularities in $E$ and $F$ respectively. We obtain some results under geometric restrictions on the sets $E$ and $F$ and we provide some examples showing the sharpness of the positive results.
\end{abstract}

\section{INTRODUCTION}

Let $O \subset \mathbb{C}$ be an open set and $S_{1}, S_{2}$ be two relatively closed subsets in $O, S=S_{1} \cup S_{2}$. The following result is due to Aronszajn [Aro35]

Theorem 1. Any function $f$ analytic in $O \backslash S$ coincides with $\left.\left(f_{1}+f_{2}\right)\right|_{(O \backslash S)}$ where $f_{j}$ are analytic in $O \backslash S_{j}, j=1,2$.

In modern textbooks this fact is treated (if at all) as a trivial example illustrating general sheaf theoretic and $\bar{\partial}$ approaches and related to the first Cousin problem; [Aro35] is never quoted (see e.g. [BG91, p. 225], [Hör90]) not to mention its famous predecessors Poincaré [Poi92] and Fréchet [Fré30] although their approaches remain interesting even now. The first version of Theorem 1 with an ingenious proof appeared in 1892 [Poi92] $\left(O=\mathbb{C}, S_{1}=[-1,1], S_{2}=\right.$ $(-\infty,-1] \cup[1,+\infty)$ which is, of course, equivalent to $\left.S_{1}=(-\infty, 0], S_{2}=[0, \infty)\right)$. The great author was motivated by his dispute with Borel concerning possible generalizations of the classical notion of analytic continuation. It is not quite clear who was right (see the discussion in [Val54, Chapter IV, section 21-22]), but the explicit (non-linear) construction of $f_{1}, f_{2}$ given in [Poi92] (exposed also in [Val54]) is very elegant (as was shown in [MH71], in the Poincaré situation there is no linear operator $f \rightarrow\left(f_{1}, f_{2}\right)$ (from $H o l(O \backslash S) \rightarrow \operatorname{Hol}\left(O \backslash S_{1}\right) \times \operatorname{Hol}\left(O \backslash S_{2}\right)$ where $\operatorname{Hol}(G)$ stands for the space of all functions analytic in an open set $G$ with the usual topology). Various aspects of the separation of singularities in the spirit of the Poincaré-Aronszajn theorem (Theorem 1) are treated in [Val54], [BG91], [Hav58], [Hav84] and [Gau98].

The present article deals with a quantitative aspect of Theorem 1 related to spaces $H^{\infty}(G)$ of functions bounded and analytic in an open set $G \subset \mathbb{C}$. Namely we are interested in the case when $f$ in Theorem 1 is bounded (i.e. $f \in H^{\infty}(O \backslash S)$ ) and ask whether $f_{1}, f_{2}$ can be made bounded as well (i.e. $\left.f_{j} \in H^{\infty}\left(O \backslash S_{j}\right), j=1,2\right)$.

Date: October 6, 2006.

The first author is partially supported by RFBR grant \#06-01-00313 and by the grant for Leading Scientific Schools \#NSH-2266-2003.1. The last author is supported by DGICYT grant MTM2005-08984-C02-02 and the CIRIT grant 2005SGR00611. 
Definition. Let $O, S_{1}, S_{2}, S$ be as in Theorem 1. We say $\left(S_{1}, S_{2}\right)$ is a bounded separation pair (bs-pair) in $O$ if any $f \in H^{\infty}(O \backslash S)$ is representable by the formula

$$
f=f_{1}+f_{2} \text { in } O \backslash S
$$

where $f_{j} \in H^{\infty}\left(O \backslash S_{j}\right)$.

The problem which is implicit in the definition can be restated as follows (just putting $G_{j}=$ $O \backslash S_{j}, G=O \backslash S$ ): given open sets $G_{1}, G_{2} \subset \mathbb{C}$ is it possible to represent an arbitrary $f \in H^{\infty}(G)$ by (1) with $f_{j} \in H^{\infty}\left(G_{j}\right), j=1,2$ ?

We want more or less efficient (preferably geometric) conditions imposed on $\left(S_{1}, S_{2}\right)$, resp $\left(G_{1}, G_{2}\right)$ and ensuring (1) for any $f \in H^{\infty}(O \backslash S)$ with $f_{j} \in H^{\infty}\left(O \backslash S_{j}\right)$ (resp. $f \in H^{\infty}\left(G_{1} \cap\right.$ $\left.\left.G_{2}\right), f_{j} \in H^{\infty}\left(G_{j}\right)\right)$.

One of the first results of this kind is due to Polyakov [Pol83]; it was ancillary in his work on bounded extensions of functions $f \in H^{\infty}(C)$ to the polydisk $\mathbb{D}^{n}$ (where $\mathbb{D}=\{|z|<1\}$ ) for an analytic curve $C \subset \mathbb{D}^{n}$; see also [HP84]. For $G_{1}=\mathbb{D} \cap\{\Im z>0\}, G_{2}=\mathbb{D} \cap\{\Re z>0\}$ and any $f \in H^{\infty}\left(G_{1} \cap G_{2}\right)$ Polyakov proved (1) in $G_{1} \cap G_{2}$ where $f_{j}$ is analytic in $G_{j}$ and bounded in $G_{j} \cap \frac{1}{2} \mathbb{D}$.

In [HN01] (whose authors were unaware of [Pol83]) and in [Hav04] the bs-pairs were investigated in a systematic way and in much more general setting. As in [Pol83], in [HN01] it was essential that $S_{1}, S_{2}$ meet transversally (a generic situation is presented below on Figure 6 (section 3.2 below); the results of [HN01] apply to more general transversal configurations). In [Hav04] a class of tangent bs-pairs was described. Turning to the present article we start with some remarks on bs-pairs.

If $S_{1}, S_{2} \subset \mathbb{C}$ are compact and disjoint, then $\left(S_{1}, S_{2}\right)$ is a bs-pair in $\mathbb{C}$ : this is an easy consequence of the Cauchy integral formula. Simple examples of pairs which are not bs in $\mathbb{C}$ (in particular the Poincaré pair $\left.S_{1}=(-\infty, 0], S_{2}=[0, \infty)\right)$ are in [HN01].

The bs-problem is related to the Alice Roth Fusion Lemma (see [Gai87], [Gam69], [Dou66], [HN01]). In particular, the usual localization technique (the Vitushkin operator) gives the following result (see [HN01]): $\left(S_{1}, S_{2}\right)$ is a bs-pair in a bounded open set $O$ if $\operatorname{dist}\left(S_{1}, S_{2}\right)>0$.

Dealing with the bs-problem for a pair $\left(S_{1}, S_{2}\right)$ we may always assume that $S_{1} \cap S_{2}=\emptyset$. (Indeed, $\left(S_{1}, S_{2}\right)$ is a bs-pair in $O$ iff $\left(S_{1} \backslash s, S_{2} \backslash s\right)$ is a bs-pair in $O \backslash s$ where $s=S_{1} \cap S_{2}$.)

A really subtle and interesting situation arises when $S_{1} \cap S_{2}=\emptyset$ and $\operatorname{dist}\left(S_{1}, S_{2}\right)=0$. Let us denote the closure of a set $E$ by Clos $E$, and put $K_{j}=\operatorname{Clos} S_{j}, j=1,2, k=K_{1} \cap K_{2}$. It is shown in [HN01] that $\left(S_{1}, S_{2}\right)$ is a bs-pair in $O$ iff $\left(S_{1} \cap v, S_{2} \cap v\right)$ is a bs-pair in O for a neighbourhood $v$ with respect to $\mathbb{C}$ of $k$. Thus only "the germs of $S_{1}$ and $S_{2}$ at $k$ " are responsible for the bs-property of $\left(S_{1}, S_{2}\right)$ in $O$.

In the present paper (as in [HN01] and [Hav04]) we concentrate on the simplest case when $k$ is a singleton.

We continue the study of "transversal" pairs $\left(S_{1}, S_{2}\right)$ meeting at a point and obtain a very general result applicable to arbitrary relatively closed parts $S_{j}$ of the upper half-plane $\mathbb{C}^{+}$separated by two rays $\eta=k \zeta, \eta=k_{1} \zeta, \zeta>0,0<\arg (k)<\arg \left(k_{1}\right)<\pi$. In [HN01] the transversally meeting sets $S_{1}$ and $S_{2}$ were supposed to be parts of certain rectifiable arcs. We believe, however, that the concrete bounded splittings $f \rightarrow\left(f_{1}, f_{2}\right)\left(f \in H^{\infty}(O \backslash S), f_{j} \in H^{\infty}\left(O \backslash S_{j}\right)\right)$ 
constructed in [HN01] are of independent interest; they are also applicable to many tangent pairs $\left(S_{1}, S_{2}\right)$, see [Hav04].

Unlike [HN01], we do not exclude the tangency of $S_{1}$ and $S_{2}$ and find rather sharp descriptions of tangent bs-pairs $\left(S_{1}, S_{2}\right)$ in $\mathbb{C}^{+}$when $k=\{0\}$; in some cases these descriptions yield necessary and sufficient conditions for a tangent pair of arcs to be bs (Theorem 7).

We mainly put $O=\mathbb{C}^{+}$; note that $\left(S_{1}, S_{2}\right)$ is a bs-pair in $\mathbb{C}^{+}$iff $\left(\phi\left(S_{1}\right), \phi\left(S_{2}\right)\right)$ is a bs-pair in $\phi\left(\mathbb{C}^{+}\right)$for a conformal homeomorphism $\phi$ of $\mathbb{C}^{+}$.

The article consists of three sections. In $\S 1$ we obtain sufficient conditions for a pair $\left(S_{1}, S_{2}\right)$ to be bs in $\mathbb{C}^{+}$(Theorem 4). Our approach is a reduction to the $\bar{\partial}$-problem

$$
\bar{\partial} u=f \bar{\partial} \chi
$$

in an open set $O$ where $f \in H^{\infty}(O \backslash S)$ is a given function to be split as in (1), and $\chi$ is a cutting factor which is $\equiv 1$ near $S_{1}$ and $\equiv 0$ near $S_{2}$ in $O$; the pair $\left(S_{1}, S_{2}\right)$ is bs in $O$ if (2) has always (for any $f$ ) a bounded solution $u$.

Theorems 2 and 3 are preparatory; they are corollaries of deep results by Berndtsson, [Ber92] on bounded solutions of the $\bar{\partial}$-problem in a disc. From them we deduce our main result of $\S 1$ (Theorem 4), treating transversal and tangent pairs $S_{1}, S_{2} \subset \mathbb{C}^{+}$with $k=\{0\}$; concrete examples are discussed in 1.4. In 1.5, we give a direct and explicit solution of (2), not using Berndtsson's results and thus making our $\S 1$ essentially self-contained. In 1.6 we give yet another very explicit proof of Theorem 4 for pairs $\left(S_{1}, S_{2}\right)$ in $\mathbb{C}^{+}$where $S_{1}, S_{2}$ are separated by a sector with vertex at the origin. In 1.7 we briefly describe quite explicit bounded splittings $f \rightarrow\left(f_{1}, f_{2}\right)$ applicable to a class of pairs $\left(S_{1}, S_{2}\right), S_{j} \subset \mathbb{C}^{+}$; the splittings of $1.5,1.7$ are linear in $f \in$ $H^{\infty}\left(\mathbb{C}^{+} \backslash S\right)$.

The objects of $\S 2$ are pairs $\left(S_{1}, S_{2}\right)$ of smooth arcs in a sector $A$ meeting tangentially at its vertex, which are not bs in $A$ (and, in fact, in any domain $O \supset A$ ).

The construction is a rather involved "condensation of singularities", an accumulation of "badly splittable" pairs of arcs in $A$ based on the Banach theorem on surjective operators. The results are different depending on whether the common tangent of $S_{1}$ and $S_{2}$ at the vertex is a side of $A$ (Theorem 6) or is strictly inside $A$ (Theorem 5). These theorems show the sharpness of the results of $\S 1$.

The results of $\S \S 1-2$ are combined in $\S 3$ to state Theorem 7 which includes, among other things, the tangential case when $S_{j}$ is the graph of a real $\mathcal{C}^{1+\varepsilon}$-function $\phi_{j}$ on $[0, b]$ such that

$$
\phi_{j}(0)=\phi_{j}^{\prime}(0)=0,0<\phi_{1}(t)<\phi_{2}(t) \quad \text { for } t \in(0, b] .
$$

It turns out that $\left(S_{1}, S_{2}\right)$ is a bs-pair in $\mathbb{C}^{+}$iff

$$
\liminf _{x \rightarrow 0} \frac{\phi_{2}(x)-\phi_{1}(x)}{\phi_{1}(x)}>0
$$

(i.e. $S_{1}$ and $S_{2}$ are hyperbollicaly separated in $\mathbb{C}^{+}$).

In section 3.2 we show that for a Jordan domain $G=G_{1} \cap G_{2}$ where the domains $G_{j}$ are Jordan as well, a function $f \in H^{\infty}(G)$ may exist which is not representable as $f_{1}+f_{2}$ with $f_{j} \in H^{\infty}\left(G_{j}\right)$. In section 3.3, we construct an example illustrating the difference between the 
case of connected sets $S_{1}, S_{2}$ (arcs) we dealt with in Theorem 7, and disconnected $S_{j}$ 's where, apparently, some new results are needed to grasp the bs-property.

\section{SOME CLASSES OF BS-PAIRS IN THE UPPER HALF-PLANE}

1.1. Reduction to a $\bar{\partial}$-problem. Let $S_{1}, S_{2}$ be disjoint relatively closed subsets of the domain $O$, and $f \in H^{\infty}(O \backslash S), S=S_{1} \cup S_{2}$. Let $U_{1}, U_{2}$ be disjoint open neighbourhoods of (resp.) $S_{1}, S_{2}$ in $O$. Consider a bounded locally Lipschitz function $\chi$ in $O$ such that

$$
\chi\left|U_{1}=0, \chi\right| U_{2}=1 .
$$

Extend $f$ to $O$ putting $f \mid S=0$. Any (distributional) solution $u$ of the $\bar{\partial}$-problem

$$
\bar{\partial} u=f \bar{\partial} \chi \quad \text { in } O
$$

is a continuous function, since $f \bar{\partial} \chi$ is locally bounded in $O$. We assume $u \in \mathcal{C}(O)$ and put

$$
f_{2}=f \chi-u, \quad f_{1}=f(1-\chi)+u \text { in } O,
$$

so that $f=f_{1}+f_{2}$ in $O$. Clearly, $f_{j}$ is continuous in $O \backslash S_{j}$ and $\bar{\partial} f_{j}=0$ in $O \backslash S_{j}$, whence it is analytic in $O \backslash S_{j}$. It is bounded if $u$ is. We arrive at the following conclusion:

if (2) admits a solution $u \in L^{\infty}(O)$ for any $f \in H^{\infty}(O \backslash S)$, then $\left(S_{1}, S_{2}\right)$ is a bs-pair with respect to $O$

1.2. bs-pairs and a result by Berndtsson. Bounded solvability of a general $\bar{\partial}$-equation

$$
\bar{\partial} u=\rho
$$

in $\mathbb{D}$ was studied in [Ber92]. The result of [Ber92] we need can be easily adapted to $\mathbb{C}^{+}=\{\Im z>$ $0\}$. By $L^{1}(E), E \subset \mathbb{C}$, we mean $L^{1}(E, d A)$ where $d A$ is the area element.

Theorem 2. Suppose that $\rho \in L^{1}\left(\mathbb{C}^{+}\right)$vanishes in $\mathbb{C}^{+} \cap\{|z|>R\}$ for a positive $R$. If

(a) $\rho d A$ is a Carleson measure in $\mathbb{C}^{+}$, and

(b) $\rho \Im z \in L^{\infty}\left(\mathbb{C}^{+}\right)$,

then the (distributional) problem (5) has a solution $u \in L^{\infty}\left(\mathbb{C}^{+}\right)$.

Combining this result with section 1.1 we get

Theorem 3. Suppose $S_{1}, S_{2} \subset \mathbb{C}^{+}$are bounded, disjoint, and relatively closed. Suppose there exists a locally Lipschitz real function $\chi$ in $\mathbb{C}^{+}$satisfying (3), vanishing in $\mathbb{C}^{+} \cap\{|z|>R\}$ and such that

(a) $|\nabla \chi| d A$ is a Carleson measure in $\mathbb{C}^{+}$,

(b) $|\nabla \chi(\zeta)| \leq C / \Im \zeta,\left(\zeta \in \mathbb{C}^{+}\right)$.

Then $\left(S_{1}, S_{2}\right)$ is a bs-pair in any domain $O \subset \mathbb{C}^{+}$containing $S$.

Proof. Note that $2|\bar{\partial} \chi|=|\nabla \chi|$. By Theorem 2 the $\bar{\partial}$-problem 2 has a bounded solution in $\mathbb{C}^{+}$ (thus in $O$ ), and section 1.1 applies. 
1.3. Sets $S_{1}, S_{2}$ separated by graphs. To get a palpable description of some bs-pairs let us assume that there are a number $\mu>0$ and a Lipschitz nonnegative function $g$ on $\mathbb{R}$ such that

$$
\begin{aligned}
& S_{1} \subset U_{1}=\left\{\xi+i \eta \in \mathbb{C}^{+}: \eta<g(\xi)\right\}, \\
& S_{2} \subset U_{2}=\left\{\xi+i \eta \in \mathbb{C}^{+}: \eta>(1+\mu) g(\xi)\right\} .
\end{aligned}
$$

Theorem 4. Suppose $S_{1}, S_{2}$ are relatively closed in $\mathbb{C}^{+}$and bounded. If (6) holds then $\left(S_{1}, S_{2}\right)$ satisfies the assumptions of Theorem 3 and thus is a bs-pair in any $O \subset \mathbb{C}^{+}$containing $S$.

Our assumption (6) means that $S_{1}, S_{2}$ are separated by the corridor $\left\{\xi+i \eta \in \mathbb{C}^{+}: g(\xi) \leq\right.$ $\eta \leq(1+\mu) g(\xi)\}$ whose hyperbolic width is positive (since for $g(\xi)>0$ the hyperbolic distance between $\xi+i g(\xi)$ and $\xi+i(1+\mu) g(\xi)$ exceeds a positive number not depending on $\xi \in \mathbb{R})$. We shall see in $\S 2$ that this condition is sharp.

Proof. Put

$$
\chi_{0}(\xi, \eta)= \begin{cases}0 & \text { if } 0<\eta<g(\xi) \\ \frac{\eta-g(\xi)}{\mu g(\xi)} & \text { if } \xi \in \mathbb{R}, 0<g(\xi) \leq \eta \leq(1+\mu) g(\xi), \\ 1 & \text { if } \eta>(1+\mu) g(\xi) .\end{cases}
$$

Thus $\chi_{0}(\xi, \eta)=1$ if $\xi \in \mathbb{R}, g(\xi)=0, \eta>0$. Clearly $\chi_{0}$ is locally Lipschitz on the set $\left\{\xi+i \eta \in \mathbb{C}^{+}: g(\xi)>0\right\}$. If $g(\xi)=0, \eta>0$, then $(1+\mu) g\left(\xi^{\prime}\right)<\eta^{\prime}$ for $\xi^{\prime} \simeq \xi, \eta^{\prime} \simeq \eta$ whence $\chi_{0}\left(\xi^{\prime}, \eta^{\prime}\right)=1$. We see that $\chi_{0} \equiv 1$ in a neighbourhood of any point $\xi+i \eta \in \mathbb{C}^{+}$with $g(\xi)=0$, and $\chi_{0}$ is locally Lipschitz in $\mathbb{C}^{+}$. By definition $\chi_{0}\left|U_{1}=0, \chi_{0}\right| U_{2}=1$. Take a large $R>0$ (so that $S \subset\{|\zeta|<R\}$ ) and then a $\mathcal{C}_{0}^{\infty}$-function $\chi_{1}$ which is real and $\equiv 1$ on $\{|\zeta|<R\}$. Then

$$
\chi=\chi_{0} \chi_{1}
$$

satisfies (3). We only have to check (a) and (b) of Theorem 3. But $\nabla \chi=0$ on $U_{1} \cup U_{2}$ and

$$
|\nabla \chi(\xi, \eta)| \leq \frac{C\left(\chi_{1}\right)}{\mu g(\xi)}\left(\frac{\eta\left|g^{\prime}(\xi)\right|}{g(\xi)}+1\right) \leq C\left(g, \mu, \chi_{1}\right) / \eta
$$

for almost all $\xi \in\{g>0\}$ and $\eta \in(g(\xi),(1+\mu) g(\xi))$ (recall that $g^{\prime} \in L^{\infty}(\mathbb{R})$ ). Thus (b), holds. To prove (a) consider a Carleson box $B=(a, b) \times(0, b-a)(a, b \in \mathbb{R}, a<b)$. We have

$$
\int_{B}|\nabla \chi| d A=\int_{B \cap\{g(\xi)<\eta<(1+\mu) g(\xi)\}}|\nabla \chi| d A \leq C \int_{a}^{b}\left(\int_{g(\xi)}^{(1+\mu) g(\xi)} \frac{d \eta}{\eta} d \xi\right)=C \log (1+\mu)(b-a) .
$$

\subsection{Examples.}

1.4.1. Example 1. For $k>0$ put $g(\xi)=k \xi(\xi \geq 0), g(\xi)=0(\xi<0)$. In this case Theorem 4 is illustrated by pairs $\left(S_{1}, S_{2}\right)$ separated by a sector $\{\xi+i \eta: \xi>0, k \xi<\eta<(1+\mu) k \xi\}$. Compared with [HNO1] this result is a progress. In [HN01] some transversally meeting bs-pairs were described with an explicit and elementary splitting formula $f=f_{1}+f_{2}\left(f \in H^{\infty}(O \backslash\right.$ $S), f_{j} \in H^{\infty}\left(O \backslash S_{j}\right)$ ). But in [HN01] certain regularity conditions were imposed on $S_{1}, S_{2}$ (in particular they had to be contained in a union of rectifiable arcs) whereas Theorem 4 allows arbitrary relatively closed sets separated by a sector. 


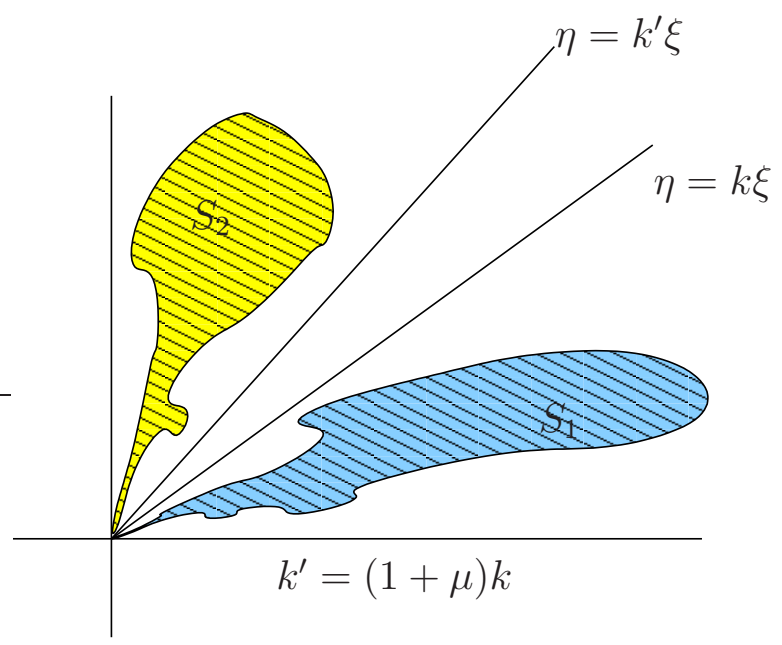

FIGURE 1. Example 1

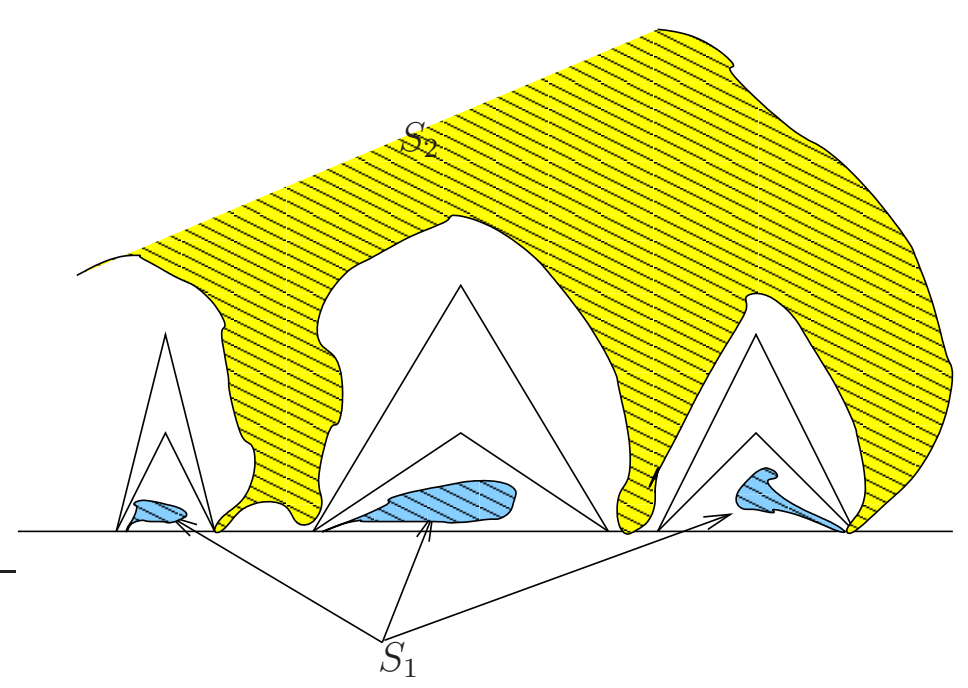

FIGURE 2. Example 2

1.4.2. Example 2. Let $G \subset \mathbb{R}$ be a bounded open set. Theorem 4 is applicable to $g(\xi)=$ $\operatorname{dist}(\xi, \mathbb{R} \backslash G)$ (see figure 2)

1.4.3. Example 3. Theorem 4 is also applicable to some tangentially meeting bs-pairs $\left(S_{1}, S_{2}\right)$. Suppose $g \in \mathcal{C}^{1}(\mathbb{R}), g \geq 0, g(0)=g^{\prime}(0)=0$. On figure $3 S_{1}$ and $S_{2}$ meet tangentially at the origin, but form a bs-pair in $\mathbb{C}^{+}$(and in any subdomain of $\mathbb{C}^{+}$containing $S=S_{1} \cup S_{2}$ ).

1.5. Theorem 2 revisited: an explicit solution. The $\bar{\partial}$-estimates that we needed included uniform bounds in the whole set $\mathbb{C}^{+}$and not only on the boundary. That is the reason that we could not use Carleson estimates, but had to appeal to the more sophisticated Theorem 2 and we had 


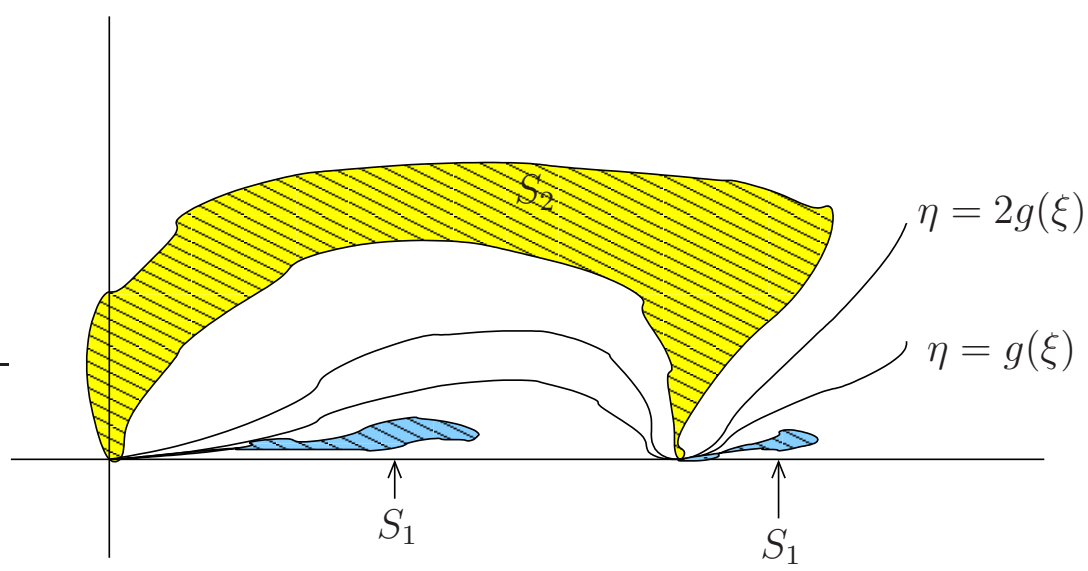

FiguRE 3. Example3

to assume some extra regularity on the data $\rho$ in (5) apart from the Carleson condition. The estimates by Berndtsson rely on some a priori estimates and therefore the solution is not explicit. Nevertheless Jones has found an explicit non-linear formula [Jon83] to solve the $\bar{\partial}$ equation that gives bounded boundary values for the solution when the data is a Carleson measure. Seip has observed (see [Sei04, Chapter1]) that this formula can be adapted to get Theorem 2. For the sake of completeness we present the adapted Jones solution.

Proof. We assume that $\rho \in L^{1}\left(\mathbb{C}^{+}\right)$and vanishes in $\mathbb{C}^{+} \cap\{|z|>R\}$ for a positive $R>0$. Furthemore we assume $|\rho| d A$ is a Carleson measure in $\mathbb{C}^{+}$and that $\rho \Im z \in L^{\infty}\left(\mathbb{C}^{+}\right)$. Set

$$
u(z)=\frac{2 i}{\pi} \int_{\Im \zeta>0} \frac{1}{z-\zeta} \frac{\Im \zeta}{z-\bar{\zeta}} \exp \left\{\alpha \int_{\Im w \leq \Im \zeta}\left(\frac{-i}{z-\bar{w}}+\frac{i}{\zeta-\bar{w}}\right)|\rho(w)| d A(w)\right\} \rho(\zeta) d A(\zeta) .
$$

The real number $\alpha$ can be chosen freely. Now, $\bar{\partial} u=\rho$. Indeed $u$ is of the form

$$
u(z)=\frac{1}{\pi} \int \frac{h(z, \zeta)}{z-\zeta} \rho(\zeta) d A(\zeta)
$$

where $h$ is continuous in $\mathbb{C}^{+} \times \mathbb{C}^{+}$, and $h(\zeta, \zeta)=1$ in the diagonal; $h(\cdot, \zeta)$ is holomorphic for any $\zeta \in \mathbb{C}^{+}$. For any test function $\phi \in \mathcal{C}_{0}^{\infty}\left(\mathbb{C}^{+}\right), \zeta \in \mathbb{C}^{+}$and small $\varepsilon>0$,

$$
\begin{aligned}
& \frac{1}{\pi} \int_{\mathbb{C}^{+} \backslash B(\zeta, \varepsilon)} \frac{h(z, \zeta)}{z-\zeta} \bar{\partial} \phi(z) d A(z)=\frac{1}{\pi} \int_{\mathbb{C}^{+} \backslash B(\zeta, \varepsilon)} \bar{\partial}\left(\frac{h(z, \zeta)}{(z-\zeta)} \phi(z)\right) d A(z)= \\
& -\frac{1}{2 \pi i} \int_{|z-\zeta|=\varepsilon} \frac{h(z, \zeta)}{z-\zeta} \phi(z) d z \rightarrow-\phi(\zeta) \quad \text { as } \varepsilon \rightarrow 0,
\end{aligned}
$$

the countour integral is taken counterclockwise. Thus

$$
\int_{\mathbb{C}^{+}} u(z) \bar{\partial} \phi(z) d A(z)=\int_{\mathbb{C}^{+}} \rho(\zeta) \frac{1}{\pi} \int_{\mathbb{C}^{+}} \frac{h(z, \zeta)}{(z-\zeta)} \bar{\partial} \phi(z) d A(z) d A(\zeta)=-\int_{\mathbb{C}^{+}} \phi \rho d A
$$


and $\bar{\partial} u=\rho$ follows. The hard part is to obtain estimates for $u$. We take

$$
\alpha^{-1}=\sup _{\Im \zeta>0} \int_{\Im w \leq \Im \zeta} \frac{2 \Im \zeta}{|w-\bar{\zeta}|^{2}}|\rho(w)| d A(w) .
$$

This supremum is finite because we assume that $|\rho| d A$ is a Carleson measure. We get $|u(z)| \leq$

$\frac{2}{\pi \alpha} \int_{\Im \zeta>0} \frac{\alpha}{|z-\zeta|} \frac{\Im \zeta}{|z-\bar{\zeta}|} \exp \left\{\alpha \int_{\Im w \leq \Im \zeta}\left(\frac{-(\Im w+\Im z)}{|z-\bar{w}|^{2}}+\frac{2 \Im \zeta}{|\zeta-\bar{w}|^{2}}\right)|\rho(w)| d A(w)\right\}|\rho(\zeta)| d A(\zeta)$.

We use the definition of $\alpha$ and we get

$$
|u(z)| \leq \frac{2 e}{\pi \alpha} \int_{\Im \zeta>0} \frac{\alpha}{|z-\zeta|} \frac{\Im \zeta}{|z-\bar{\zeta}|} \exp \left\{\alpha \int_{\Im w \leq \Im \zeta} \frac{-(\Im(w)+\Im(z))}{|z-\bar{w}|^{2}}|\rho(w)| d A(w)\right\}|\rho(\zeta)| d A(\zeta) .
$$

We then write

$$
|u(z)| \leq I_{1}+I_{2},
$$

where

$I_{1}=\frac{2 e}{\alpha \pi} \int_{2|z-\zeta| \geq \Im z} \frac{\alpha}{|z-\zeta|} \frac{\Im \zeta}{|z-\bar{\zeta}|} \exp \left\{\alpha \int_{\Im w \leq \Im \zeta} \frac{-(\Im(w)+\Im(z))}{|z-\bar{w}|^{2}}|\rho(w)| d A(w)\right\}|\rho(\zeta)| d A(\zeta)$

and

$I_{2}=\frac{2 e}{\alpha \pi} \int_{2|z-\zeta|<\Im z} \frac{\alpha}{|z-\zeta|} \frac{\Im \zeta}{|z-\bar{\zeta}|} \exp \left\{\alpha \int_{\Im w \leq \Im \zeta} \frac{-(\Im(w)+\Im(z))}{|z-\bar{w}|^{2}}|\rho(w)| d A(w)\right\}|\rho(\zeta)| d A(\zeta)$.

When $2|z-\zeta| \geq \Im z$, then $|z-\zeta| \geq|z-\bar{\zeta}| / 5$ and thus $I_{1}$ is bounded by a constant times

$$
\frac{1}{\alpha} \int_{\Im \zeta>0} \frac{\alpha \Im \zeta}{|z-\bar{\zeta}|^{2}} \exp \left\{\alpha \int_{\Im w \leq \Im \zeta} \frac{-\Im(w)}{|z-\bar{w}|^{2}}|\rho(w)| d A(w)\right\}|\rho(\zeta)| d A(\zeta) .
$$

If $f$ is any positive function defined on $t \geq 0$ and $R_{0}=\int_{0}^{\infty} f(t) d t$ (which may eventually be $\infty)$, then

$$
\int_{0}^{\infty} f(r) \exp \left(-\int_{0}^{r} f(t) d t\right) d r=\int_{0}^{R_{0}} e^{-t} d t \leq 1
$$

We apply this inequality to the function

$$
f(t)=\int_{\mathbb{R}} \frac{\alpha t}{|z-(x-i t)|^{2}}|\rho(x+i t)| d x
$$

and we get that (8) is bounded by $\alpha^{-1}$.

On the other hand $I_{2}$ is easy to estimate if we drop the exponential term and use the fact that $|\rho(z)| \leq C / \Im z$. 
1.6. An explicit solution of (2) for transversally meeting sets $S_{1}, S_{2} \subset \mathbb{C}^{+}$. Here we return to Example 1 of section 1.4 and give another very simple solution of the problem

$$
\bar{\partial} u=\rho, \rho=f \bar{\partial} \chi,
$$

where $\chi($ see (7)) corresponds to $g$ which is zero on $(-\infty, 0]$ and is linear on $[0, \infty): g(\xi)=k \xi$, $\xi \geq 0$, for a $k>0$. The sets $S_{1}, S_{2}$ are separated by the sector

$$
A_{k, \mu}=\{(\xi, \eta): k \xi \leq \eta \leq(1+\mu) k \xi\},
$$

and $\partial \chi$ is supported by a sector $T=A_{k, \mu} \cap\{|z|<R\}$. Put $u=C^{\rho}-a$, where $C^{\rho}=-\frac{1}{\pi} \rho \star \frac{1}{z}$ is the standard solution of (9) and

$$
a(\zeta)=\frac{1}{\pi} \int_{\mathbb{C}^{+}} \frac{\bar{z}}{z} \frac{\rho(z)}{\zeta-\bar{z}} d A(z), \quad \zeta \in \mathbb{C}^{+} .
$$

Note that $\rho \equiv 0$ off $T$, and $|\rho(z)| \leq C / \Im z \simeq 1 /|z|$ for $z \in T$ whence $\rho \in L^{1}\left(\mathbb{C}^{+}, d A\right)$, and functions $C^{\rho}, a$ make sense.

Clearly, $\bar{\partial} u=\rho$, since $a$ is analytic in $\mathbb{C}^{+}$, and we only have to prove that $u$ is bounded in $\mathbb{C}^{+}$. We have

$$
u(\zeta)=\frac{2 i \zeta}{\pi} \int_{T} \frac{\Im z \bar{\partial} \chi(z) f(z) d A(z)}{z(\zeta-z)(\zeta-\bar{z})},
$$

whence

$$
|u(\zeta)| \leq c|\zeta| \int_{T} \frac{d A(z)}{|z||\zeta-z||\zeta-\bar{z}|}=C|\zeta| J(\zeta), \quad \zeta \in \mathbb{C}^{+}
$$

Fix a small $q>0$ (to be specified later). Then

$$
J(\zeta) \leq \int_{T \cap\{|z-\zeta|<q|\zeta|\}}+\int_{T \cap\{|z-\zeta|>q|\zeta|,|z|>q|\zeta|\}}+\int_{T \cap\{|z-\zeta|>q|\zeta|,|z|<q|\zeta|\}}=I+I I+I I I .
$$

Now, $|z| \simeq \Im z$ for $z \in T$. Estimating I we may write $|z| \geq|\zeta|-|z-\zeta| \geq(1-q)|\zeta|$,

$$
|\bar{z}-\zeta| \geq 2 \Im z-|z-\zeta| \geq c|z|-q|\zeta| \geq c^{\prime}|\zeta|
$$

where $c=c(k, \mu)>0, c^{\prime}=c(1-q)-q>0$ if $q$ is small, and

$$
I \leq \frac{c_{1}}{|\zeta|^{2}} \int_{|z-\zeta|<q|\zeta|} \frac{d A(z)}{|\zeta-z|}=\frac{c_{2}}{|\zeta|}
$$

The Hölder inequality (with $p=3, q=3 / 2$ ) and $|\bar{z}-\zeta| \geq|z-\zeta|$ give

$$
I I \leq \int_{|z-\zeta|>q|\zeta|,|z|>q|\zeta|} \frac{d A(z)}{|z||z-\zeta|^{2}} \leq\left(\int_{|z|>q|\zeta|} \frac{d A(z)}{|z|^{3}}\right)^{1 / 3}\left(\int_{|z-\zeta|>q|\zeta|} \frac{d A(z)}{|z-\zeta|^{3}}\right)^{2 / 3}=\frac{c_{3}}{|\zeta|} .
$$

At last,

$$
I I I \leq \int_{|z-\zeta|>q|\zeta|,|z|<q|\zeta|} \frac{d A(z)}{|z||z-\zeta|^{2}} \leq \frac{1}{q^{2}|\zeta|^{2}} \int_{|z|<q(|\zeta|} \frac{d A(z)}{|z|}=\frac{c_{4}}{|\zeta|} .
$$

Combining (10) with (11)-(13) we see that $u$ is bounded. 
1.7. Another explicit and bounded solution to equation (2). Here we again exploit the special form of the right side of (2) (recall that Theorem 2 is aimed at general right sides $\rho \in L^{1}\left(\mathbb{C}^{+}\right)$, but we only deal with $\rho=f \bar{\partial} \chi$, where $f \in H^{\infty}\left(\mathbb{C}^{+} \backslash S\right)$ and $\chi$ is a smooth cutting factor).

This time we consider a function $g: \mathbb{R} \rightarrow[0,+\infty)$ and assume $g \in \mathcal{C}^{1+\varepsilon}(\mathbb{R}), \varepsilon>0, g \equiv 0$ on $(-\infty, 0], g(x)>0$ for $x>0$ (in Theorem $4 g$ was a Lipschitz function and it could vanish at some positive points). The functions $\chi_{0}, \chi_{1}, \chi$ are the same as in Theorem 4. Fix a small $b>0$ so that $\chi_{1} \equiv 1$ on the corridor

$$
G_{b}=\{\xi+i \eta: 0<\xi<b, g(\xi)<\eta<(1+\mu) g(\xi)\}
$$

and $\chi_{0} \equiv \chi$ on $G_{b}$. For $t \in[1,1+\mu]$ put $\gamma_{t}(\xi)=\xi+i \operatorname{tg}(\xi), 0 \leq \xi \leq b$.

Suppose $f$ is as in Theorem $4\left(f \in L^{\infty}\left(\mathbb{C}^{+}\right), f \mid S=0, f\right.$ is analytic in $\left.\mathbb{C}^{+} \backslash S\right)$. Put

$$
u(\zeta)=\frac{1}{\pi} \int_{\mathbb{C}^{+}} \frac{f(z) \bar{\partial} \chi(z)}{\zeta-z} d A(z)+\frac{\mu}{2 \pi i} \int_{\bar{\gamma}_{1}} \frac{f(\bar{z}) d z}{\zeta-z}, \zeta \in \mathbb{C}^{+} .
$$

Then $u$ is bounded in $\mathbb{C}^{+}$and satisfies (2).

Recall that $\gamma_{1}$ is the path $t \rightarrow t+i g(t), t \in[0, b]$, so that the path $\bar{\gamma}_{1}$ (i.e. the reflected graph of $g$ ) does not intersect $\mathbb{C}^{+}$, and the contour integral is analytic in $\mathbb{C}^{+}$. At the same time the double integral represents "the standard solution of (2)" (note that $\bar{\partial} \chi$ is summable in $G_{b}$, see the last estimate in Section 1.3). Thus our $u$ satisfies (2) in $\mathbb{C}^{+}$, and the only problem is its boundedness. The standard solution may blow up at the origin, but its growth is counterbalanced by the contour integral. A detailed proof will be given in [HO06].

Our definition of $u$ combined with Section 1.1 generates a linear operator $f \rightarrow\left(f_{1}, f_{2}\right)$ splitting $f \in H^{\infty}\left(\mathbb{C}^{+} \backslash S\right)$ into the sum of functions $f_{j} \in H^{\infty}\left(\mathbb{C}^{+} \backslash S_{j}\right), j=1,2$.

\section{NegAtive Results}

In this section we describe some pairs $\left(S_{1}, S_{2}\right)$ of subsets of a sector $A$ which are not bs-pairs in $A$ (Theorems 5, 6 in section 2.5.3 and 2.6). Combined with the results of $\S 1$ they give some necessary and sufficient geometric conditions for two smooth graphs to form a bs-pair in $\mathbb{C}^{+}$ (see $\S 3$ ). We start with preliminary technical results on "badly splittable" pairs of arcs $K_{1}, K_{2}$ in a domain $O$ which means the existence of $\phi \in H^{\infty}(O \backslash K), K=K_{1} \cup K_{2}$ with $|\phi| \leq 1$ in $O \backslash K$ and such that a representation $\phi=\phi_{1}+\phi_{2}, \phi_{j} \in H^{\infty}(O \backslash K)$, is only possible with a very big $\sup \left\{\left|\phi_{1}(\zeta)\right|: \zeta \in O \backslash K_{1}\right\}$. If $S_{1}, S_{2} \subset O$ contain the elements $K_{1}, K_{2}$ of arbitrarily badly splittable pairs $\left(K_{1}, K_{2}\right)$ (i.e. $\left.S_{j} \supset K_{j}, j=1,2\right)$, then $\left(S_{1}, S_{2}\right)$ is not a bs-pair in $O$. These vague considerations are made precise in sections 2.1-2.4, and then applied to quite concrete Theorems 5, 6 .

2.1. Cells and their rotundities. A cell is by definition a pair $(g, A)$ where $g$ is a Jordan domain with a rectifiable boundary $\partial g$, and $A$, "the center" of $g$, is a point in $g$. Sometimes we write $g$ instead of $(g, A)$. Put

$$
\rho(g)(=\rho((g, A)))=\frac{2 \pi \operatorname{dist}(A, \partial g)}{l(\partial g)}
$$


where $l(\partial g)$ denotes the length of $\partial g$. Clearly, $\rho(g) \leq 1$, a geometrically obvious fact (an instant proof is given by the formula $\left.1=(2 \pi i)^{-1} \int_{\partial g} \frac{d z}{z-A}\right)$; the equality $\rho(g)=1$ occurs iff $g$ is a disc centered at $A$. We call $\rho(g)$ the rotundity of the cell $g(=(g, A))$.

2.2. Functions at a large distance from $H^{\infty}(g)$ in a cell $g$. Let $K$ be a compact subset of $g$ containing $A$. We assume $A$ to be a boundary point of $K$ (in our applications $K$ will be just a simple arc)

Lemma 1. Let $\phi$ be a function analytic in $\mathbb{C} \backslash K, \phi(\infty)=0$. Then any $h \in H^{\infty}(g)$ satisfies

$$
\|\phi-h\|_{g \backslash K} \geq \frac{\rho(g)}{2} \limsup _{A}|\phi|
$$

where $\|\cdot\|_{E}$ denotes $\|\cdot\|_{\infty, E}$.

In other words, if $\phi$ is very big near $A$ and $(g, A)$ is sufficiently rotund, then any $h \in H^{\infty}(g)$ is far away from $\phi$ in $H^{\infty}(g \backslash K)$.

Proof. Any $h \in H^{\infty}(g)$ has the angular boundary value $h(g)$ at almost every $q \in \partial g$, and

$$
h(p) \equiv \frac{1}{2 \pi i} \oint_{\partial g} \frac{h(q)}{q-p} d q \quad \text { for } p \in g .
$$

Moreover, $\oint_{\partial g} \frac{\phi(q)}{q-p} \equiv 0$ for $p \in g$. Thus putting $\|\cdot\|=\|\cdot\|_{g \backslash K}$, we have

$$
\begin{array}{r}
|\phi(p)| \leq|h(p)|+\|\phi-h\|= \\
\frac{1}{2 \pi}\left|\oint_{\partial g} \frac{h(q)-\phi(q)}{q-p} d q\right|+\|\phi-h\| \leq \\
\left(\frac{1}{\rho(g)}+1\right)\|\phi-h\| \leq \frac{2}{\rho(g)}\|\phi-h\| .
\end{array}
$$

Letting $p \rightarrow A$ we get (14).

2.3. Pairs not admitting bounded separation in a domain (an abstract scheme). Lemma 1 suggests a method to construct pairs $\left(S_{1}, S_{2}\right)$ which are not bs in a domain $O$.

2.3.1. Suppose $S_{1}, S_{2}$ are relatively closed disjoint and nowhere dense parts of a domain $O$. Suppose there is a number $C$ such that for any (big) $M>0$ there exists a cell $g(=(g, A))$, $g \subset O, A \in K_{1}$, and a pair $\left(K_{1}, K_{2}\right)$ of compact sets such that $K_{j} \subset S_{j}, j=1,2$, and $K_{1} \subset g$, $\rho(g) \geq C^{-1}$, and for a pair $\left(\phi_{1}, \phi_{2}\right)$ of functions analytic, respectively, in $\mathbb{C} \backslash K_{j}, j=1,2$, we have

$$
\phi_{1}(\infty)=\phi_{2}(\infty)=0,\left|\phi_{1}+\phi_{2}\right| \leq C \text { in } \mathbb{C} \backslash K, K=K_{1} \cup K_{2},
$$

whereas

$$
\limsup _{A}\left|\phi_{1}\right|>M
$$

Lemma 2. If $S_{1}, S_{2}$ and $O$ enjoy property 2.3.1, then $\left(S_{1}, S_{2}\right)$ is not a bs-pair in $O$.

Note that $\phi_{j} \in H^{\infty}\left(\mathbb{C} \backslash K_{j}\right), j=1,2$, since $K_{1}, K_{2}$ are disjoint. 
Proof. Put $S=S_{1} \cup S_{2}$. If $\left(S_{1}, S_{2}\right)$ were a bs-pair in $O$, then the operator $\left(f_{1}, f_{2}\right) \rightarrow\left(f_{1}+\right.$ $\left.f_{2}\right)(O \backslash S)$ from $\left.H^{\infty}\left(O \backslash S_{1}\right) \times H^{\infty}\left(O \backslash S_{2}\right)\right)$ to $H^{\infty}(O \backslash S)$ would be surjective, and, by the Banach open mapping theorem, we could find a number $N>0$ such that any $f \in H^{\infty}(O \backslash S)$ would admit a splitting

$$
f=f_{1}+f_{2} \quad \text { in } O \backslash S
$$

with $f_{j} \in H^{\infty}\left(O \backslash S_{j}\right),\left\|f_{j}\right\|_{O \backslash S_{j}} \leq N\|f\|_{O \backslash S}$.

But 2.3.1 forbids the existence of such number $N$. Indeed, let $K_{j} \phi_{j}$ be as in 2.3.1 where a big $M=M(N)$ will be specified later. Put $f_{j}^{0}=\phi_{j} \mid\left(O \backslash K_{j}\right), j=1,2$, and $f=f_{1}^{0}+f_{2}^{0}$ in $O \backslash K$, $K=K_{1} \cup K_{2}$. Then for any representation (16) of $f$ we get

$$
\begin{aligned}
& f_{1}=f_{1}^{0}-h \text { in } O \backslash S_{1} \\
& f_{2}=f_{2}^{0}+h \text { in } O \backslash S_{2}
\end{aligned}
$$

where $h \in H^{\infty}(O)$, since $f_{1}^{0}-f_{1}$ and $f_{2}-f_{2}^{0}$ are mutual analytic continuations from $O \backslash S$ across $S_{2}$ and $S_{1}$. But $f_{1}$ is in fact defined and analytic not only on $O \backslash S_{1}$, but in $O \backslash K_{1}$, since $f_{1}^{0} \in H^{\infty}\left(O \backslash K_{1}\right), h \in H^{\infty}(O)$. Moreover, $\left\|f_{1}\right\|_{O \backslash K_{1}}=\left\|f_{1}\right\|_{O \backslash S_{1}}$, since $S_{1}$ has no interior points. Now since $h \in H^{\infty}(g)$ and $\rho(g) \geq C^{-1}$, by Lemma 1 and (15) we have

$$
\left\|f_{1}\right\|_{O \backslash S_{1}}=\left\|f_{1}\right\|_{O \backslash K_{1}} \geq \frac{M}{2 C}
$$

For an arbitrary $N>0$ take $M>2 N C^{2}$. We get a contradiction: given $N>0$, no splitting (16) of $f$ with $f_{j} \in H^{\infty}\left(O \backslash S_{j}\right)$ satisfies $\left\|f_{1}\right\|_{O \backslash S_{1}} \leq N\|f\|_{O \backslash S}$ (recall that $\|f\|_{O \backslash S} \leq C$ ).

2.3.2. Now we are ready to describe a general scheme of constructing triples $\left(O, S_{1}, S_{2}\right)$ where $O$ is a domain, $S_{j}$ its relatively closed and disjoint subsets such that $\left(S_{1}, S_{2}\right)$ is not a bs-pair in $O$. Namely, suppose we have constructed two families $\left(K_{1}^{x}\right)_{x \in E},\left(K_{2}^{x}\right)_{x \in E}$ of compact simple arcs and two families $\left(\phi_{1}^{x}\right)_{x \in E},\left(\phi_{2}^{x}\right)_{x \in E}$ of functions in, respectively, $H^{\infty}\left(\mathbb{C} \backslash K_{j}^{x}\right), j=1,2$, and a family of points $\left(A^{x}\right)_{x \in E}$ such that
(a) $K_{1}^{x} \cap K_{2}^{x}=\emptyset$;
(b) $\phi_{j}^{x}(\infty)=0, j=1,2$;
(c) $\sup _{x \in E}\left\|\phi_{1}^{x}+\phi_{2}^{x}\right\|_{\mathbb{C} \backslash K^{x}}<+\infty, K^{x}:=K_{1}^{x} \cup K_{2}^{x}$;
(d) $A^{x} \in K_{1}^{x}$ and $\sup _{x \in E} \lim \sup _{A^{x}}\left|\phi_{1}^{x}\right|=+\infty$.

The last element of our construction is a family $\left(g^{x}, A^{x}\right)_{x \in E}$ of cells such that

(e) $K_{1}^{x} \subset g^{x}, x \in E$, and $g^{x}$ are uniformly rotund (i.e. $\inf _{x \in E} \rho\left(g^{x}\right)>0$ ).

Any family of quintuples $\left(K_{1}^{x}, K_{2}^{x}, \phi_{1}^{x}, \phi_{2}^{x}, g^{x}\right)$ enjoying (a)-(e) generates plenty of "bad" triples $\left(O, S_{1}, S_{2}\right)$. Indeed, for any domain $O$ containing $\cup_{x \in E}\left(g^{x} \cup K_{2}^{x}\right)$, any pair $\left(S_{1}, S_{2}\right)$ of its disjoint relatively closed and nowhere dense subsets is not a bs-pair with respect to $O$ provided

$$
S_{1} \supset \cup_{x \in E} K_{1}^{x}, \quad S_{2} \supset \cup_{x \in E} K_{2}^{x} .
$$

This is an immediate consequence of Lemma 2.

Sections 2.1-2.3 are an abridged version of $\S 1$ in [Hav04]. But the technique of section 2.4 is quite different from [Hav04] and results in removing a logarithmic factor from Theorem 4 of [Hav04]; our Theorem 7 is also based on this improvement. 


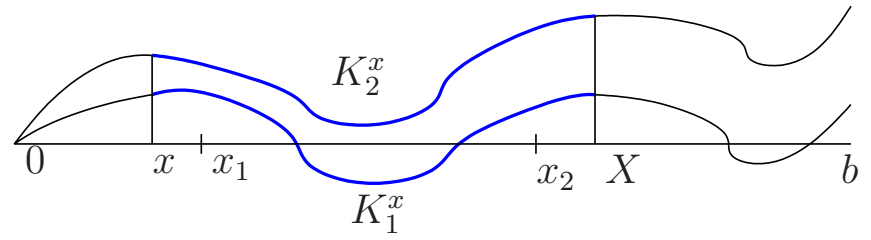

FiguRE 4. $x_{1}-x=X-x_{2}=h, X=X(x), x \rightarrow 0$

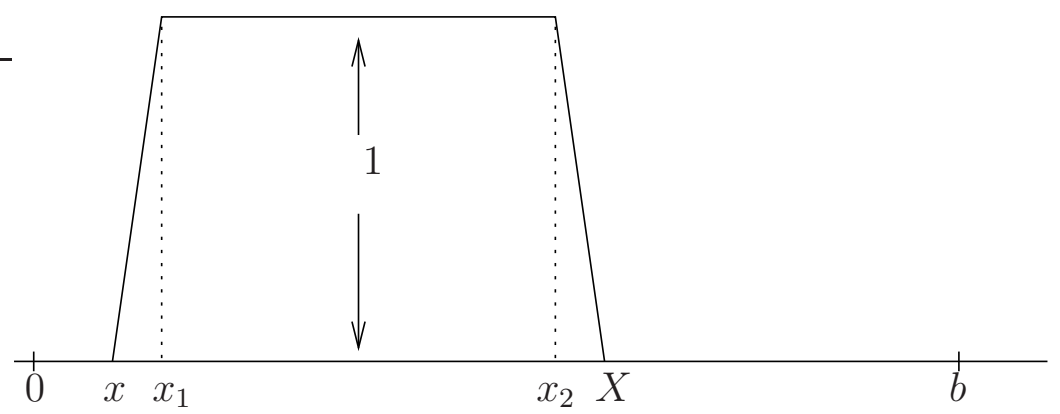

FIGURE 5. $x_{1}-x=X-x_{2}=h(x)$

2.4. A realization of the general scheme: pairs of graphs. In what follows the $\operatorname{arcs} K_{j}^{x}$ will be pieces of the graphs of real functions $\phi_{j}, j=1,2$ defined on $[0, b]$. We assume

$$
\varphi_{j} \in \mathcal{C}^{1+\varepsilon}([0, b]), \varphi_{j}(0)=\varphi_{j}^{\prime}(0)=0, j=1,2, \varphi_{1}(t)<\varphi_{2}(t) \text { for } t \in(0, b] .
$$

It will be also convenient to assume

$$
\left|\varphi_{j}^{\prime}(t)\right|<1 / 2, t \in[0, b]
$$

(this assumption doesn't affect the generality).

\subsubsection{We put}

$$
K_{j}^{x}=\left\{t+i \varphi_{j}(t): x \leq t \leq X\right\}, x \in(0, b]
$$

The choice of $X=X(x)$ will be specified later.

To define the functions $\phi_{j}^{x}$ (see (c) on page 12) we need a piecewise linear function $f^{x}$ defined on $\mathbb{R}$ as shown on Figure 5 , so that $f^{x} \equiv 0$ on $\mathbb{R} \backslash(x, X), f^{x} \equiv 1$ on $\left[x_{1}, x_{2}\right], f^{x}$ is linear on $\left[x, x_{1}\right],\left[x_{2}, X\right]$; the positive number $h=h(x)$ will be chosen later. Now put

$$
\tilde{f}_{j}^{x}(\zeta)=f^{x}(\Re \zeta) \quad(\zeta \in \mathbb{C}) .
$$

Define the functions $\phi_{j}^{x}$ by the formulas

$$
\begin{aligned}
\phi_{j}^{x}(\zeta)= & (-1)^{j-1} \frac{1}{2 \pi i} \int_{K_{j}} \frac{\tilde{f}_{j}^{x}(z)}{z-\zeta} d z= \\
& (-1)^{j-1} \frac{1}{2 \pi i} \int_{x}^{X} \frac{f^{x}(t)\left(1+i \varphi_{j}^{\prime}(t)\right)}{(t-\xi)+i\left(\varphi_{j}(t)-\eta\right)} d t, \quad \zeta=\xi+i \eta \in \mathbb{C} \backslash K_{j}^{x} .
\end{aligned}
$$


The first (contour) integral in (20) is oriented from the left to the right.

In fact the integral (20) can be easily evaluated, so that $\phi_{j}^{x}$ become explicit linear combinations of some elementary functions of $\zeta$ (compositions of $\zeta \log \zeta$ and $\log \zeta$ with some Möbius functions). But we prefer to keep the integral representation of $\phi_{j}^{x}$, the estimates of their explicit versions not being any shorter. Getting rid of integration makes it possible to apply the scheme of Section 2.3 to general continua $S_{1}, S_{2}$ (not just curves), see [Hav04]; but it also makes the description of bs-pairs of graphs not as precise as our Theorem 7.

Put $A^{x}(=A)=x+i \varphi_{1}(x)$. Functions $\phi_{j}^{x}$ belong to $H^{\infty}\left(\mathbb{C} \backslash K_{j}^{x}\right)$ and vanish at infinity; $\phi_{j}^{x}$ is continuous at both sides of the arc $K_{j}^{x}$. In particular, $\lim _{A_{x}} \phi_{1}^{x}$ exists and coincides with $\frac{1}{2 \pi i} \int_{K_{1}^{x}} \frac{\tilde{f}_{1}^{x}(z) d z}{z-A^{x}}$. We denote it by $\phi_{1}(A)$.

We are going to show that $\left|\phi_{1}(A)\right|$ is big if $h<<X-x$. Indeed,

$$
\begin{gathered}
\phi_{1}(A)=\left(\int_{x}^{x_{1}}+\int_{x_{1}}^{x_{2}}+\int_{x_{2}}^{X}\right) \frac{f(t)}{2 \pi i} \frac{\left(1+i \varphi_{1}^{\prime}(t)\right) d t}{(t-x)+i\left(\varphi_{1}(t)-\varphi_{1}(x)\right)}=I+I I+I I I, \\
|I| \leq \frac{1}{2 \pi} \int_{x}^{x+h} \frac{t-x}{h} \frac{3 / 2 d t}{t-x}<1 .
\end{gathered}
$$

To estimate III note that any $t \in\left(x_{2}, X\right)$ satisfies $X-t<t-x$, and

$$
|I I I| \leq \frac{1}{2 \pi} \int_{X-h}^{X} \frac{X-t}{h} \frac{3 / 2 d t}{t-x}<1 .
$$

Now,

$$
|I I|=\frac{1}{2 \pi}\left|\int_{x_{1}}^{x_{2}} \frac{d\left(t+i \varphi_{1}(t)\right)}{\left(t+i \varphi_{1}(t)\right)-A}\right| \geq \frac{1}{2 \pi}\left|\log \frac{\left|\left(x_{2}-x\right)+i\left(\varphi_{1}\left(x_{2}\right)-\varphi_{1}(x)\right)\right|}{\left|\left(x_{1}-x\right)+i\left(\varphi_{1}\left(x_{1}\right)-\varphi_{1}(x)\right)\right|}\right| .
$$

The numerator of the last fraction is greater than or equal to $x_{2}-x$, the denominator is less than or equal to $3 / 2\left(x_{1}-x\right)$ whence $|I I| \geq \frac{1}{2 \pi} \log \frac{x_{2}-x_{1}}{2 h}$. But $h /\left(x_{2}-x_{1}\right)=(h /(X-x))(1-$ $2 h /(X-x))^{-1}$, so that

$$
\lim _{x \rightarrow 0}\left|\phi_{1}^{x}\left(A^{x}\right)\right|=+\infty \text { if } \lim _{x \rightarrow 0} \frac{h(x)}{x-X(x)}=0 .
$$

\subsection{Upper estimates of $\left|\phi_{1}^{x}+\phi_{2}^{x}\right|$. Put}

$$
K^{x}=K_{1}^{x} \cup K_{2}^{x}, \phi^{x}=\phi_{1}^{x}+\phi_{2}^{x} .
$$

(Sometimes we omit the index $x$ ). We now have to concentrate on the upper estimate of $|\phi|$ in $\mathbb{C} \backslash K$ (see (c) on page 12). We may apply the elementary maximum modulus principle to $\phi$, since $\phi$ is continuous up to the sides of the arcs $K_{1}, K_{2}$. Expressing the boundary values of $\phi$ on $K$ by the Sohotsky-Plemelj formulas and using the estimate $|f| \leq 1$, we reduce our problem to the upper estimate of $\left|J\left(z_{0}\right)\right|$ where

$$
J\left(z_{0}\right)=p \cdot v \cdot \int_{K} \tilde{f}(z) \frac{d z}{z-z_{0}}, \quad z_{0} \in K,
$$


$K_{1}$ is oriented from the left to the right, $K_{2}$ is oriented in the opposite direction, so that, hopefully, the contributions of $K_{1}$ and $K_{2}$ will be mutually tempered. Put

$$
z_{j}(t)=t+i \varphi_{j}(t), \quad t \in[0, b],
$$

and suppose

$$
z_{0} \in K_{1}, z_{0}=z_{1}\left(t_{0}\right), t_{0} \in[0, b] .
$$

(the case $z_{0} \in K_{2}$ is symmetric). We have

$$
J\left(z_{0}\right)=p \cdot v \cdot \int_{x}^{X} f(t) \mathcal{K}\left(t, z_{0}\right) d t
$$

where

$$
\mathcal{K}\left(t, z_{0}\right)=\frac{z_{1}^{\prime}(t)}{z_{1}(t)-z_{0}}-\frac{z_{2}^{\prime}(t)}{z_{2}(t)-z_{0}} .
$$

For a smooth function $\lambda$ of a real variable $t$ put

$$
R_{t_{0}}(\lambda)(t)=\lambda(t)-\lambda\left(t_{0}\right)-\lambda^{\prime}(t)\left(t-t_{0}\right) .
$$

Put $\Delta=\varphi_{2}-\varphi_{1}$. Using this notation the reader can check the following identity:

$$
\mathcal{K}\left(t, z_{0}\right)=\mathcal{K}_{1}\left(t, z_{0}\right)+\mathcal{K}_{2}\left(t, z_{0}\right)
$$

where

$$
\begin{aligned}
& \mathcal{K}_{1}\left(t, z_{0}\right)=\frac{i R(\Delta)+\varphi_{2}^{\prime}(t) R\left(\varphi_{1}\right)-\varphi_{1}^{\prime}(t) R\left(\varphi_{2}\right)}{\left(z_{1}(t)-z_{0}\right)\left(z_{2}(t)-z_{0}\right)}, \\
& \mathcal{K}_{2}\left(t, z_{0}\right)=\frac{i \Delta\left(t_{0}\right) z_{1}^{\prime}(t)}{\left(z_{1}(t)-z_{0}\right)\left(z_{2}(t)-z_{0}\right)}, R=R_{t_{0}} .
\end{aligned}
$$

An easy estimate of the kernel $\mathcal{K}_{1}$ follows from the inequalities

$$
\left|z_{j}(t)-z_{0}\right| \geq\left|t-t_{0}\right|,\left|R\left(\varphi_{j}\right)(t)\right| \leq c\left(\varphi_{j}\right)\left|t-t_{0}\right|^{1+\varepsilon}, j=1,2,
$$

since $\varphi_{j}($ and $\Delta)$ are in $\mathcal{C}^{1+\varepsilon}$; thus, for $t_{0} \in[0, b]$,

$$
\left|p . v . \int_{x}^{X} f(t) \mathcal{K}_{1}\left(t, z_{0}\right) d t\right| \leq c \int_{x}^{X} \frac{d t}{\left|t-t_{0}\right|^{1-\varepsilon}} \leq c^{\prime}
$$

where $c, c^{\prime}$ depend only on $\varphi_{1}, \varphi_{2}$ and $\varepsilon$ (but not on $z_{0}$ ) for any $f$ satisfying $|f(t)| \leq 1$ on $[0, b]$.

Turn now to

$$
J_{2}=p . v \cdot \int_{x}^{X} \mathcal{K}_{2}\left(t, z_{0}\right) f(t) d t=\int_{[x, X] \backslash a}+\int_{a}=j_{1}+j_{2}
$$

where $a=[x, X] \cap\left[t_{0}-\Delta\left(t_{0}\right), t_{0}+\Delta\left(t_{0}\right)\right]$. The integral $j_{1}$ is easy (recall that $|f| \leq 1$, $\left.\left|\varphi_{1}^{\prime}\right| \leq 1 / 2\right)$ :

$$
\left|j_{1}\right| \leq 2 \Delta\left(t_{0}\right) \int_{\left|t-t_{0}\right|>\Delta\left(t_{0}\right)} \frac{d t}{\left(t-t_{0}\right)^{2}}=4
$$


But $j_{2}$, unlike preceding estimates, requires some special properties of $f$ and additional restrictions on the proximity of $K_{1}$ and $K_{2}$ (i.e. smallness restrictions on $\Delta$ ). To estimate $j_{2}$ put $F(t)=f(t) /\left(z_{2}(t)-z_{0}\right)$; we get

$$
\begin{aligned}
j_{2}= & i \Delta\left(t_{0}\right) p \cdot v \cdot \int_{a} F(t) \frac{z_{1}^{\prime}(t) d t}{z_{1}(t)-z_{0}}= \\
& i \Delta\left(t_{0}\right) \int_{a} \frac{F(t)-F\left(t_{0}\right)}{z_{1}(t)-z_{1}\left(t_{0}\right)} d z_{1}(t)+i \Delta\left(t_{0}\right) F\left(t_{0}\right) p \cdot v \cdot \int_{K_{1}^{a}} \frac{d z}{z-z_{0}}=I+I I,
\end{aligned}
$$

where $K_{1}^{a}=\left\{t+i \varphi_{1}(t): t \in a\right\}$. Now,

$$
\begin{gathered}
|I| \leq \Delta\left(t_{0}\right) \int_{a}\left|\frac{F(t)-F\left(t_{0}\right)}{t-t_{0}}\right|\left(1+\left|\varphi_{1}^{\prime}(t)\right|\right) d t \leq 2 \Delta\left(t_{0}\right)\left\|F^{\prime}\right\|_{\infty, a}|a|, \\
\left|F^{\prime}(t)\right| \leq\left|f^{\prime}(t)\right| /\left|z_{2}(t)-z_{1}(t)\right|+|f(t)| \frac{1+\left|\varphi_{2}^{\prime}(t)\right|}{\left|z_{2}(t)-z_{1}\left(t_{0}\right)\right|^{2}} .
\end{gathered}
$$

But

$$
\left|z_{2}(t)-z_{1}\left(t_{0}\right)\right| \geq\left|\varphi_{2}(t)-\varphi_{1}\left(t_{0}\right)\right| \geq \Delta\left(t_{0}\right)-\left\|\varphi_{2}^{\prime}\right\|_{\infty}\left|t-t_{0}\right| \geq \Delta\left(t_{0}\right) / 2,
$$

since $\left\|\varphi_{2}^{\prime}\right\|<1 / 2, t \in a$. From (28), (29) and the estimates $\|f\|_{\infty} \leq 1,\left\|f^{\prime}\right\|_{\infty} \leq 1 / h$, $|a| \leq 2 \Delta\left(t_{0}\right)$ we conclude that

if

$$
|I| \leq 2 \Delta\left(t_{0}\right)\left(\frac{2}{h \Delta\left(t_{0}\right)}+\frac{8}{\Delta\left(t_{0}\right)^{2}}\right) 2 \Delta\left(t_{0}\right)=\frac{8 \Delta\left(t_{0}\right)}{h}+32 \leq 40,
$$

$$
\Delta\left(t_{0}\right) \leq h, \quad \text { for any } t_{0} \in[x, X],
$$

an important restriction expressing the proximity of $\varphi_{1}$ and $\varphi_{2}$ (note that $h<<X-x$ by (21)).

We are left now with the integral II (see (27)). From $\left|F\left(t_{0}\right)\right| \Delta\left(t_{0}\right)=f\left(t_{0}\right)$ we conclude

$$
\begin{aligned}
& |I I| \leq f\left(t_{0}\right)\left|p \cdot v \cdot \int_{K_{1}^{a}} \frac{d z}{z-z_{0}}\right|, \\
& \quad a=[x, X] \cap\left[t_{0}-\Delta\left(t_{0}\right), t_{0}+\Delta\left(t_{0}\right)\right], K_{1}^{a}=K_{1} \cap\{\zeta: \Re \zeta \in a\}, t_{0} \in[x, X] .
\end{aligned}
$$

2.5.1. We have to consider two cases:

Case 1: $x \leq t_{0}-\Delta\left(t_{0}\right) \leq t_{0}+\Delta\left(t_{0}\right) \leq X$;

Case 2: $t_{0}-\Delta(t)<x$ or $t_{0}+\Delta\left(t_{0}\right)>X$.

In Case $1 a=\left[t_{0}-\Delta\left(t_{0}\right), t_{0}+\Delta\left(t_{0}\right)\right]$, and

$$
\begin{aligned}
|I I| & \leq\left|p \cdot v \cdot \int_{K_{1}^{a}} \frac{d z}{z-z_{0}}\right|=\left|\log \frac{z_{1}\left(t_{0}+\Delta\left(t_{0}\right)\right)-z_{1}\left(t_{0}\right)}{z_{1}\left(t_{0}-\Delta\left(t_{0}\right)\right)-z_{1}\left(t_{0}\right)}\right| \leq \\
& \leq\left|\log \frac{\left|z_{1}\left(t_{0}+\Delta\left(t_{0}\right)\right)-z_{1}\left(t_{0}\right)\right|}{\left|z_{1}\left(t_{0}-\Delta\left(t_{0}\right)\right)-z_{1}\left(t_{0}\right)\right|}\right|+2 \pi= \\
& =\frac{1}{2}\left|\log \frac{\left(\Delta\left(t_{0}\right)\right)^{2}+\left(\varphi_{1}\left(t_{0}+\Delta\left(t_{0}\right)\right)-\varphi_{1}\left(t_{0}\right)\right)^{2}}{\left(\Delta\left(t_{0}\right)\right)^{2}+\left(\varphi_{1}\left(t_{0}-\Delta\left(t_{0}\right)\right)-\varphi_{1}\left(t_{0}\right)\right)^{2}}\right|+2 \pi \leq B,
\end{aligned}
$$


$B$ being an absolute constant, since $\left|\varphi_{1}\left(t_{0} \pm \Delta\left(t_{0}\right)\right)-\varphi_{1}\left(t_{0}\right)\right| \leq \Delta\left(t_{0}\right) / 2$ whence the fraction under the logarithm is in $(4 / 5,5 / 4)$.

In Case 2 we suppose, e.g., that $t_{0}-\Delta\left(t_{0}\right)<x$ (the case $X<t_{0}+\Delta\left(t_{0}\right)$ is symmetric). Then $0 \leq t_{0}-x<\Delta\left(t_{0}\right) \leq h$ by (30), so that $t_{0} \in\left[x, x_{1}\right]$ and $f\left(t_{0}\right)=\left(t_{0}-x\right) / h$. Now $a=\left[x, t_{0}+\Delta\left(t_{0}\right)\right]$ (note that $t_{0}+\Delta\left(t_{0}\right)>X$ is impossible, because this inequality implies $2 \Delta\left(t_{0}\right)=\left(t_{0}+\Delta\left(t_{0}\right)\right)-\left(t_{0}-\Delta\left(t_{0}\right) \geq X-x>2 h\right.$, a contradiction with (30)). Thus,

$$
|I I| \leq \frac{t_{0}-x}{h}\left[\left|\log \frac{\left|z_{1}\left(t_{0}+\Delta\left(t_{0}\right)\right)-z_{1}\left(t_{0}\right)\right|}{\left|z_{1}(x)-z_{1}\left(t_{0}\right)\right|}\right|+2 \pi\right]
$$

The numerator of the last fraction is between $\Delta\left(t_{0}\right)$ and $2 \Delta\left(t_{0}\right)$ (since $\left|\varphi_{1}^{\prime}\right|<1 / 2$ ), its denominator is between $t_{0}-x$ and $2\left(t_{0}-x\right)$ so that, by (30) and (31),

$$
|I I| \leq \frac{t_{0}-x}{\Delta\left(t_{0}\right)}\left[\log \frac{\Delta\left(t_{0}\right)}{t_{0}-x}+\log 2+2 \pi\right] \leq e^{-1}+\log 2+2 \pi
$$

(since $y|\log y| \leq e^{-1}$ for $\left.y \in(0,1]\right)$.

Summing up, our functions $\phi_{1}^{x}, \phi_{2}^{x}$ satisfy conditions (a)-(d) of Section 2.3.2, if (21) and (30) are fulfilled.

2.5.2. We now have to specify $h(x), X(x)$ to satisfy (21) and (30), and then construct the cells $g^{x}$ centered at $A^{x}=x+i \varphi_{1}(x)$ and satisfying condition (e) in 2.3.2. These definitions will be given twice, one time for the sector $A_{k}=\{\zeta=\xi+i \eta \in \mathbb{C}: \xi>0,|\eta|<k \xi\}$, and the second for its upper half $A_{k}^{+}=A_{k} \cap \mathbb{C}^{+}$.

For any $R>0$ we may assume the graphs of $\varphi_{1}$ and $\varphi_{2}$ are in $A_{k}$, replacing $b$ by a smaller number if needed. Put

$$
\epsilon(x)=\sup \left\{\frac{\left|\varphi_{1}(t)\right|+\left|\varphi_{2}(t)\right|}{t}: 0<t \leq 2 x\right\}, X(x)=2 x, h(x)=2 \varepsilon(x) x .
$$

Then $\lim _{x \rightarrow 0} \frac{h(x)}{X(x)-x}=\lim _{x \rightarrow 0} 2 \epsilon(x)=0$ (see (17) in section 2.4), and we get (21). If $t \in$ $[x, X(x)]$, then $\Delta(t)=\varphi_{2}(t)-\varphi_{1}(t) \leq\left|\varphi_{2}(t)\right|+\left|\varphi_{1}(t)\right| \leq \epsilon(x) t \leq h(x)$, so that (30) holds. Put $g^{x}=A_{k} \cap\{\zeta \in \mathbb{C}: \Re \zeta<3 x\}$. The inclusion $K_{1}^{x} \subset g^{x}$ is obvious if $x>0$ is small (again by (17) and (19) in section 2.4) as is the uniform rotundity of $g^{x}$ with respect to the center $A^{x}$ (at this point $A_{k}$ cannot be replaced by $A_{k}^{+}$, since the center $A^{x}$ may be too close to the boundary of $A_{k}^{+}$and the rotundity of $g^{x}$ be very small).

2.5.3. We have arrived at the first result of this section.

Let $S_{1}, S_{2}$ be the graphs of $\varphi_{j} \mid(0, b], j=1,2$ where $\varphi_{j}$ are as in section 2.4, see (17). Without loss of generality (see [HN01] or the Introduction) we may assume $A_{k} \supset S=S_{1} \cup S_{2}$. Suppose $S \subset\{|\zeta|<R\}(=R \mathbb{D})$.

Theorem 5. For any $k>0$ and any domain $O \supset A_{k} \cap R \mathbb{D},\left(S_{1}, S_{2}\right)$ is not a bs-pair in $O$.

The proof follows from Lemma 2, since the families $\left(\phi_{j}^{x}\right),\left(K_{j}^{x}\right), j=1,2,\left(g^{x}\right)$ enjoying properties (a)-(e) have been constructed in sections 2.4.1-2.5.2. 
2.6. Two graphs in $A_{k}^{+}$. We still assume $S_{1}, S_{2}, \varphi_{1}, \varphi_{2}$ are as in section 2.4 (see (17)), but we also suppose

$$
\varphi_{1}(t)>0, t \in(0, b]
$$

Taking $b<b(k)$ we may assume $S \subset A_{k}^{+}$. This time we have to impose a special proximity conditions on $S_{1}, S_{2}((17)$ is not sufficient to apply Lemma 2). Namely, we assume

$$
\liminf _{x \rightarrow 0} \frac{\Delta(x)}{\varphi_{1}(x)}=0
$$

Let $E \subset(0, b]$ be a set with a limit point at the origin and such that

$$
\lim _{x \rightarrow 0, x \in E} \frac{\Delta(x)}{\varphi_{1}(x)}=0 .
$$

We define $h(x), X(x)$ (and thus $K_{j}^{x}, j=1,2$ ) for $x \in E$ only:

$$
X(x)=x+\frac{1}{2} \varphi_{1}(x), h(x)=2 \varphi_{1}(x) \epsilon(x)
$$

where

$$
\epsilon(x)=\frac{\Delta(x)}{\varphi_{1}(x)}+\sup \left\{\left|\Delta^{\prime}(t)\right|: 0 \leq t \leq X(x)\right\}, x \in E,
$$

so that $\lim _{x \rightarrow 0, x \in E} h(x) /(X-x)=\lim _{x \rightarrow 0, x \in E} \varepsilon(x)=0$, and we get (21). Now, if $t \in[x, X]$, then, for a $c \in[x, t], \Delta(t)=\Delta(x)+\Delta^{\prime}(c)(t-x) \leq \epsilon(x) \varphi_{1}(x)+\epsilon(x) \varphi_{1}(x)=h(x)$ which is (30). The cell $g^{x}$ will be defined for $x \in E$ as the square $\left(x-\varphi_{1}(x), x+\varphi_{1}(x)\right) \times\left(0,2 \varphi_{1}(x)\right)$ with the center $x+i \varphi_{1}(x)$. This cell is uniformly rotund. Moreover, $K_{1}^{x} \subset g^{x}(x \in E)$. Indeed, for $t \in\left[x, x+\varphi_{1}(x)\right]$ we have $\varphi_{1}(t)=\varphi_{1}(x)+\varphi^{\prime}(c)(t-x)<2 \varphi_{1}(x)$, since we may assume $\left|\varphi_{1}^{\prime}(c)\right|<1$. Moreover, $g^{x} \subset A_{k}^{+}$for any small $x \in E$, since $\lim _{x \rightarrow 0} \frac{2 \varphi_{1}(x)}{x-\varphi_{1}(x)}=0$.

As in section 2.5.2 we get the following result.

Theorem 6. If $S \subset A_{k}^{+} \cap R \mathbb{D}$, and $\varphi_{1}, \varphi_{2}$ satisfy (17) and (33), then, for any domain $O \supset$ $A_{k}^{+} ; \cap R \mathbb{D},\left(S_{1}, S_{2}\right)$ is not a bs-pair in $O$.

\section{CONCLUDING REMARKS}

3.1. A complete description of some bs-pairs of arcs in $\mathbb{C}^{+}$. Let $\gamma_{j}:[0,1] \rightarrow \mathbb{C}, j=1,2$, be two simple $\mathcal{C}^{1+\varepsilon}$-arcs in $\mathbb{C}$ such that $\gamma_{1}(0)=\gamma_{2}(0)=0, \gamma_{1}(t) \neq \gamma_{2}(t)$ for $t \in(0,1]$. We also assume $\Im \gamma_{j}(t)>0$ for $t \in(0,1], \gamma_{j}^{\prime}(t) \neq 0$ for $t \in[0,1]$.

In this section we define $S_{j}$ as the trajectory of $\gamma_{j} \backslash\{0\}, j=1,2$. Combining Theorem 4 with Theorems 5 and 6 we obtain a complete and very clear description of all bs-pairs of this sort in $\mathbb{C}^{+}$.

Denote by $\tau_{j}$ the unit tangent vector of $\gamma_{j}$ at the origin. Note that $\Im \tau_{j} \geq 0$, since $S_{j} \subset \mathbb{C}^{+}$.

Theorem 7. I. If $\tau_{1} \neq \tau_{2}$, then $\left(S_{1}, S_{2}\right)$ is a bs-pair in $\mathbb{C}^{+}$.

II. Suppose $\tau_{1}=\tau_{2}$. Then

(a) $\left(S_{1}, S_{2}\right)$ is not a bs-pair in $\mathbb{C}^{+}$, if $\Im \tau_{1}>0$. 


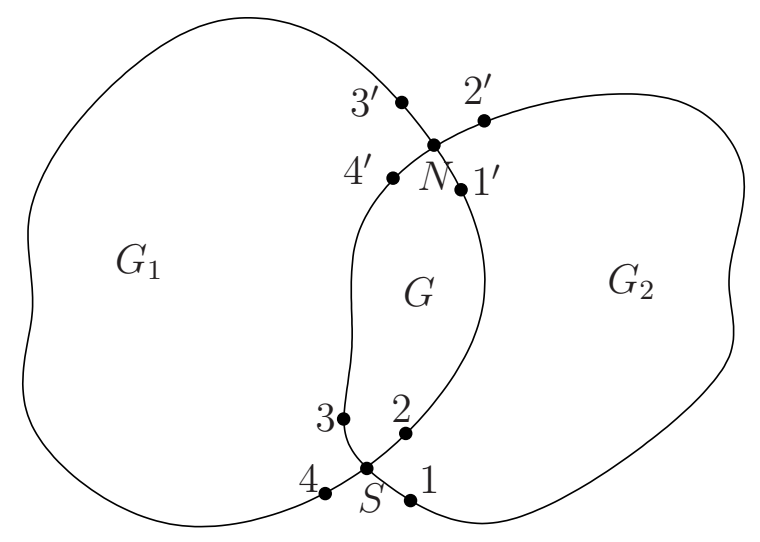

FIGURE 6

(b) If $\tau_{1}$ is real we may assume that $\tau_{1}=1$, and for a small $b>0$ the arcs $S_{j}^{b}=S_{j} \cap\{\Re \zeta<b\}$ are graphs (over $(0, b])$ of real functions $\varphi_{j} \in \mathcal{C}^{1+\varepsilon}([0, b])$ as in Theorem 5 (see (17)), and $\left(S_{1}, S_{2}\right)$ is a bs-pair in $\mathbb{C}^{+}$if, and only if, $\liminf \inf _{x \rightarrow 0} \frac{\Delta(x)}{\varphi_{1}(x)}>0, \Delta=\left|\varphi_{1}-\varphi_{2}\right|$.

Proof. In case I the pieces $S_{1}^{b}, S_{2}^{b}$ are separated by two rays in $\mathbb{C}^{+}$emanating from the origin, and we may apply Theorem 4 of $\S 1$ (see also the "transversal" example 1 in section 1.4 and the construction of section 1.6). In case II(a) $S_{1}^{b}$ and $S_{2}^{b}$ stay in a sector in $\mathbb{C}^{+}$whose bisector is parallel to $\tau_{1}$, and Theorem 5 applies. In case II(b) if (33) holds, then $\left(S_{1}, S_{2}\right)$ is not a bs-pair in $\mathbb{C}^{+}$by Theorem 6 , since $S_{1}^{b} \cup S_{2}^{b}$ is covered by a sector $A_{k}^{+}$; if (33) does not hold, then, taking $g=\left(\varphi_{1}+\varphi_{2}\right) / 2$ and a small $\mu>0$ (depending on $\left.\lim \sup _{0} \Delta / \varphi_{1}\right), S_{1}^{b}$ and $S_{2}^{b}$ are separated by the graphs of $g$ and $(1+\mu) g$, and Theorem 4 applies; see example 3 in section 1.4.

3.2. Bounded splittings of functions analytic in the intersection of Jordan domains. Consider two Jordan domains $G_{1}, G_{2}$ as on Figure 6 and their intersection $G$. Suppose the curves $\partial G_{1}, \partial G_{2}$ are piecewise $\mathcal{C}^{1}$-smooth and intersect transversally at $S$ and $N$ (i.e. any pair of arcs $1,2,3,4$ (respectively $\left.1^{\prime}, 2^{\prime}, 3^{\prime}, 4^{\prime}\right)$ meet under a positive angle at $S$, respectively at $N$ ). It is shown in [HN01, Example 4.1, section 4.6] that for any $f \in H^{\infty}(G)$ there exist functions $f_{j} \in H^{\infty}\left(G_{j}\right)$, $j=1,2$, such that

$$
f=f_{1}+f_{2} \quad \text { in } G .
$$

Now we are going to show that the transversality assumption cannot be dropped. Let $G_{1}, G_{2}$ be the Jordan domains on Figure 7, bounded respectively, by the loops $A \circ D E F A, o F D C B o$, so that $\partial G=o D E o$. Suppose $b>0$ is small, and $\partial G \cap\{0 \leq \Im z \leq b\}=o M_{1} \cup o M_{2}$ where the compact arcs $o M_{1}, o M_{2}$ are as on Figure 7, so that $\left(o M_{1}, o M_{2}\right)$ is not a bs-pair with respect to $\mathbb{C}^{+}$(see Theorem 7). Then there exists a function $f \in H^{\infty}(G)$ which cannot be split as in (34).

Proof. Take an arbitrary $F \in H^{\infty}\left(\mathbb{C}^{+} \backslash\left(o M_{1} \cup o M_{2}\right)\right)$ and put $f=F \mid G$. Suppose $f$ can be represented by (34) $\left(f_{j} \in H^{\infty}\left(G_{j}\right), j=1,2\right)$; then we prove

$$
F=F_{1}+F_{2} \quad \text { in } \mathbb{C}^{+} \backslash\left(o M_{1} \cup o M_{2}\right)
$$




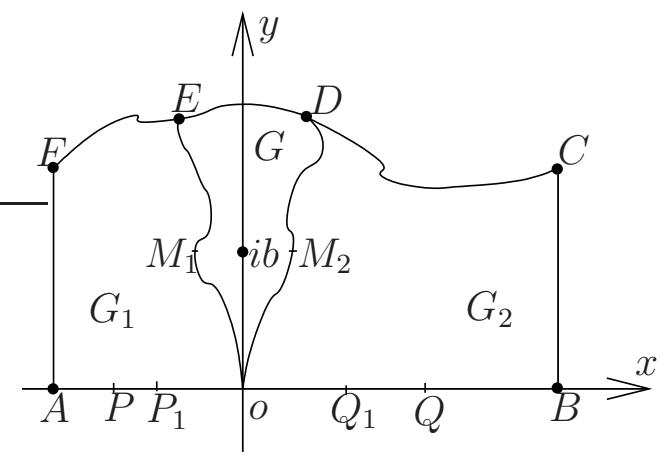

FIGURE 7. $\partial G_{1}=A o D E F A, \partial G_{2}=o E D C B o$

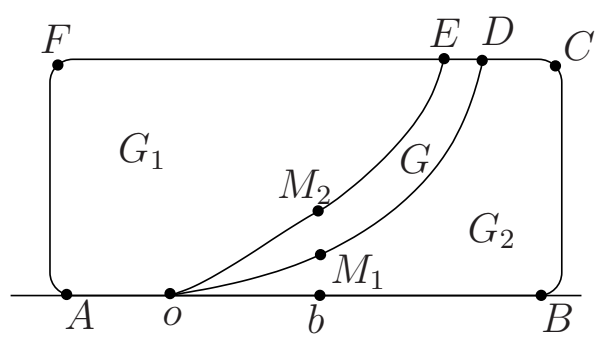

Figure 8. $\partial G_{1}=A o D E F A, \partial G_{2}=o E D C B o$

with $F_{j} \in H^{\infty}\left(\mathbb{C}^{+} \backslash o M_{j}\right), j=1,2$, a contradiction.

To deduce (35) from (34) put $H=G_{1} \cup G_{2}$ (so that $\partial H=A o B C D E F A$ ) and note that $f_{j}$ extends analytically from $G_{j}$ to $H \backslash o M_{j}, j=1,2$. Indeed, $f_{1}=f-f_{2}$ in $G$ whence $f-f_{2}$ is the analytic continuation of $f_{1}$ from $G$ to $H \backslash o M_{1}$; the same argument applies to $f_{2}$. Extend $f_{1}, f_{2}$ to $(H \cup \partial H)^{\prime}=\mathbb{C} \backslash(H \cup \partial H)$ putting

$$
f_{1}(\zeta)=f_{s}(\zeta)=0, \zeta \in(H \cup \partial H)^{\prime},
$$

thus making $f_{j} \in H^{\infty}\left(\left(\partial H \cup o M_{j}\right)^{\prime}\right), j=1,2$. Applying the preseparation Corollary 3.3 from [HN01] we split $f_{1}, f_{2}$ in their domains as follows:

$$
f_{j}=\varphi_{j}+r_{j}, j=1,2
$$

where $\left.\varphi_{j} \in H^{\infty}\left([P, Q] \cup o M_{j}\right)^{\prime}\right), r_{j} \in H^{\infty}\left(\left(\partial H \backslash\left[P_{1}, Q_{1}\right]\right)^{\prime}\right)$ (see Figure 7). The identity

$$
F-\varphi_{1}-\varphi_{2}=r_{1}+r_{2} \quad \text { in } H \backslash\left(o M_{1} \cup o M_{2}\right)
$$

(which is (34)) shows that $r_{1}+r_{2}$ coincides in $H$ with a function $h \in H^{\infty}\left(\mathbb{C}^{+}\right)$, since the left side of (36) is analytic at any point of $\mathbb{C}^{+} \backslash H$, and $F, \varphi_{1}, \varphi_{2}$ are bounded in their domains. Therefore (35) holds with $F_{1}=\varphi_{1}, F_{2}=\varphi_{2}+h$.

We could get a similar example for the domains $G_{1}, G_{2}, G$ as on Figure 8. The arcs $o M_{2}$, $o M_{2}$ are the graphs of functions $\varphi_{1}, \varphi_{2} \in \mathcal{C}^{1}([0, b])$ satisfying $\varphi_{j}(0)=\varphi_{j}^{\prime}(0)=0, j=1,2$, $0<\varphi_{1}(t)<\varphi_{2}(t)(t \in(0, b]), \lim _{t \rightarrow 0} \frac{\varphi_{2}(t)-\varphi_{1}(t)}{\varphi_{1}(t)}=0$ (see Theorem 7). However this kind 


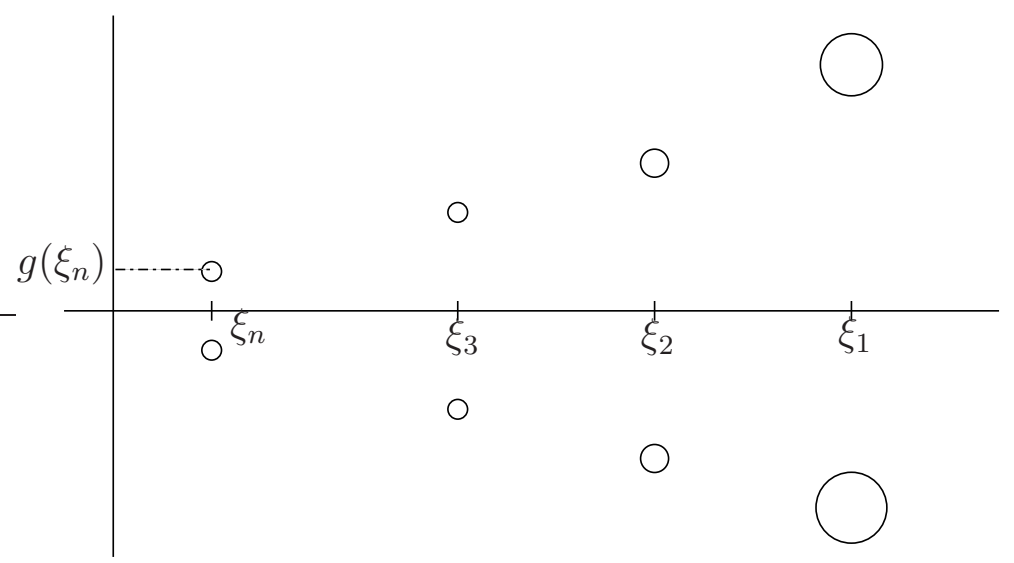

FIGURE 9

of tangency (when $o M_{1}, o M_{2}$ are tangent to $\partial H$ at the origin) is compatible with the splitting formula (34) for any $f \in H^{\infty}(G)$ if $\lim _{t \rightarrow 0} \frac{\varphi_{2}(t)-\varphi_{1}(t)}{\varphi_{1}(t)}>0$. This happens, for example, for

$$
G_{1}=\left\{0<x<1,0<y<2 x^{2}\right\}, \quad G_{2}=\left\{0<x<1, x^{2}<y<2\right\} .
$$

3.3. bs-pairs of hyperbolically close sets. Very satisfactory looking Theorem 7 deals with pairs of graphs and cannot be applied to disconnected sets. In this section we describe examples of bs-pairs $\left(S_{+}, S_{-}\right)$with respect to the right half plane $\Pi$ with $S_{+}$and $S_{-}$hyperbolically very close.

Let $g$ be a non-negative function on $[0, b]$ such that $g(\xi) \leq \xi(\xi \in[0, b])$ and $g(\xi)>0$ for $\xi \in(0, b]$. Consider a strictly decreasing sequence $\left(\xi_{n}\right)_{n=1}^{\infty}$ in $(0, b]$ tending to zero and put $\zeta_{n}=\xi_{n}+i g\left(\xi_{n}\right)$. For $r>0$ denote by $B_{n}(r)$ the closed disc $\left\{\left|\zeta-\zeta_{n}\right| \leq r\right\}$. Suppose a sequence $\left(r_{n}\right)_{n=1}^{\infty}$ of positive numbers satisfies

$$
\sum_{n=1}^{\infty} \frac{r_{n}}{g\left(\xi_{n}\right)}<\infty,
$$

so that $\sum r_{n} / \xi_{n}<\infty$ as well. We assume $r_{n}<g\left(\xi_{n}\right)$ for all $n$ and $r_{n}<\frac{1}{2} \min \left(\xi_{n}-\xi_{n-1}, \xi_{n+1}-\right.$ $\left.\xi_{n}\right)$, so that the discs $B_{n}=B_{n}\left(r_{n}\right)$ are disjoint and stay in $\mathbb{C}^{+}$. Put

$$
S_{+}=\cup_{n=1}^{\infty} B_{n}, \quad S_{-}=\cup_{n=1}^{\infty} \overline{B_{n}}, \quad S=S_{+} \cup S_{-} .
$$

Theorem 8. $\left(S_{+}, S_{-}\right)$is a bs-pair with respect to the right half plane $\Pi$.

Note that the hyperbolic distance between $\xi_{n}$ and $\bar{\xi}_{n}$ (and $B_{n}$ and $\overline{B_{n}}$ ) in $\Pi$ is comparable with $g\left(\xi_{n}\right) / \xi_{n}$ and tends to zero, if say, $g^{\prime}(0)=0$.

Proof. Any $f \in H^{\infty}(\Pi \backslash S)$ has angular boundary values a.e. on $i \mathbb{R}$ and $\partial S$, and can be represented as follows:

$$
f=f_{1}+f_{2}=f_{1}+f_{+}+f_{-} \quad \text { in } \Pi
$$


where

$$
f_{1}=-(1+z) C_{i \mathbb{R}}^{f /(1+z)}, \quad f_{+}=(1+z) \sum_{n=1}^{\infty} C_{\partial B_{n}}^{f /(1+z)}, \quad f_{-}=(1+z) \sum_{n=1}^{\infty} C_{\partial \bar{B}_{n}}^{f /(1+z)} .
$$

$\left(C_{A}^{k}\right.$ is the Cauchy type integral $\left.\frac{1}{2 \pi i} \int_{A} \frac{k(z)}{z-\zeta} d z, \zeta \notin A\right)$. The imaginary axis is oriented "upwards", and the circles $\partial B_{n}, \partial \overline{B_{n}}$ are oriented clockwise. The proof of (38) is standard $\left(f(i y) /\left(1+y^{2}\right) \in\right.$ $\left.L^{1}(\mathbb{R}, d y), f(z) /(1+z)=O(1 / z),|z| \rightarrow \infty\right)$. Now, $f_{ \pm}=(1+z) C^{\mu_{ \pm}}$where $\mu_{ \pm}$are finite complex charges on $S_{ \pm}$, so that $f_{2}$ is defined and analytic in $\mathbb{C} \backslash(S \cup\{0\})$ and $f_{2}(z)=O(1)$ $(|z| \rightarrow \infty)$. Clearly $f_{1}=f-f_{2}$ is analytic in $\Pi$, and $f_{1}(z)=O(1)(|z| \rightarrow \infty)$.

We are going to prove that

$$
f_{1} \in H^{\infty}(\Pi)
$$

whence $f_{2} \in H^{\infty}(\Pi \backslash S)$. At last we prove $f_{ \pm}$are bounded in $\Pi \backslash S_{ \pm}$and get the final splitting of $f$ into the sum of elements of $H^{\infty}\left(\Pi \backslash S_{ \pm}\right)$:

$$
f=\left(f_{1}+f_{+}\right)-f_{-} .
$$

(a) $f_{+}$is bounded on any set $\mathbb{C} \backslash A_{k}^{+}, k>2$, where $A_{k}^{+}=\{\xi+i \eta$ : $\xi>0,0<\eta<k \xi\}$ (according to our assumptions $S_{+} \subset A_{2}$ ). Indeed,

$$
\left|C_{\partial B_{n}}^{f}(\zeta)\right| \leq\|f\|_{\infty} r_{n} / \operatorname{dist}\left(\zeta, B_{n}\right), \quad \zeta \notin S_{+} .
$$

If $\Re \zeta \leq 0$, then $\operatorname{dist}\left(\zeta, B_{n}\right) \geq \xi_{n}-r_{n} \geq g\left(\xi_{n}\right)-r_{n}$; if $\Im \zeta \leq 0$, then $\operatorname{dist}\left(\zeta, B_{n}\right) \geq g\left(\xi_{n}\right)-r_{n}$, and if $\zeta \in \Pi \backslash A_{k}^{+}, \Im \zeta>0$, then

$$
\operatorname{dist}\left(\zeta, B_{n}\right) \geq\left|\zeta-\zeta_{n}\right|-r_{n} \geq\left|\zeta_{n}\right| \sin \varphi-r_{n} \geq g\left(\xi_{n}\right) \sin \varphi-r_{n},
$$

where $\varphi$ is the sector in $\Pi$ with sides $\eta=2 \xi, \eta=k \xi$. Thus, for a $c_{k}>0$,

$$
\operatorname{dist}\left(\zeta, B_{n}\right) \geq c_{k} g\left(\xi_{n}\right)
$$

for all $\zeta \in \mathbb{C}^{+} \backslash A_{k}^{+}$and $n=1,2, \ldots$. Combining (40), (41) and (37) we see that $f_{+}$is analytic and bounded in $\mathbb{C} \backslash A_{k}^{+}, k>2$. The same proof applies to $f_{-}$, so that $f_{-}$is bounded in $\mathbb{C} \backslash \overline{A_{k}^{+}}$, $k>2$. Putting $A_{k}=A_{k}^{+} \cup \overline{A_{k}^{+}} \cup(0,+\infty)$ we conclude that $f_{2}$ is bounded in $\mathbb{C} \backslash A_{k}$, and $f_{1}=f-f_{2}$ is bounded in $\Pi \backslash A_{k}$. But $f_{1}(\zeta)=O(1)(|\zeta| \rightarrow \infty, \zeta \in \Pi)$, and we may fix a large $R>0$ making $f_{1}$ bounded in $\Pi \backslash s$ where $s=A_{k} \cap\{|\zeta|<R\}$ (we fix a $k>2$, say $k=3$ ). It remains to estimate $f_{1}$ in $s$. Being analytic in $s, f_{1}$ is bounded on its sides and the arc $A_{k} \cap\{|\zeta|=R\}$ whereas

$$
\left|f_{1}(\zeta)\right|=O(\log |\zeta|),|\zeta| \rightarrow 0, \zeta \in s
$$

and (a very weak form of) Phragmen-Lindelöf applies whence $f_{1}$ is bounded in s, and (39) holds. (To get (42) write (using $|\eta|<k \xi$ )

$$
\begin{aligned}
\left|f_{1}(\zeta)\right| & \lesssim \int_{-\infty}^{+\infty} \frac{d y}{(1+|y|)(\xi+|\eta-y|)} \lesssim \int_{|y|<2 k \xi}+\int_{|y|>2 k \xi}= \\
& =O(1)+O\left(\int_{2 k \xi}^{+\infty} \frac{d y}{(1+y) y}\right)=O(|\log \xi|)=O(|\log \zeta|),
\end{aligned}
$$


since $|\zeta| \simeq \xi$ for $\zeta \in s)$.

(b) From (a) we see that $f_{2}=f_{+}+f_{-}$is bounded in $\Pi \backslash S$ (and, in fact, in $\mathbb{C} \backslash(S \cup\{0\})$ ). We already know $f_{+}$is bounded in $\mathbb{C} \backslash\left(A_{k}^{+} \cup\{0\}\right)$. We only have to show $f_{+}$is bounded in $A_{k}^{+} \backslash S_{+}$. But in $A_{k}^{+} \backslash S_{+}$

$$
f_{+}=f-f_{1}-f_{-}
$$

$f$ is bounded in $\Pi \backslash S, f_{1}$ is bounded in $\Pi$ (see (39)) and we have proved $f_{-}$is bounded in $\mathbb{C}^{+}$. Thus $f_{+} \in H^{\infty}\left(A_{k}^{+} \backslash S_{+}\right), f_{+} \in H^{\infty}\left(\mathbb{C} \backslash\left(S^{+} \cup\{0\}\right)\right)$. The boundedness of $f_{-}$in $\Pi \backslash S_{-}$follows now from $f_{-}=f_{2}-f_{+}$.

\section{REFERENCES}

[Aro35] N. Aronszajn, Sur les décompositioins des fonctions analytiques uniformes et sur leur applications, Acta Math. 65 (1935), 1-156.

[Ber92] B. Berndtsson, Weighted estimates for $\bar{\partial}$ in $\mathbb{C}$, Duke Math. J. 66 (1992), 239-255.

[BG91] C. A. Berenstein and R. Gay, Complex variables, an introduction, Graduate Texts in Mathematics, vol. 125, Springer-Verlag, New York, N.Y., 1991.

[Dou66] A. Douady, Le problème des modules pour les sous-espaces analytiques compacts d'un espace analytique donné, Contemporary Problems in Theory Anal. Functions (internat. Conf., Erevan, 1965) (Russian), Izdat. "Nauka", Moscow, 1966, pp. 141-143. MR 35 \#1820

[Fré30] M. Fréchet, Sur certaines décompositions de la fonction complexe uniforme la plus générale, Acta Math. 54 (1930), 37-79.

[Gai87] D. Gaier, Lectures on complex approximation, Birkhäuser Boston Inc., Boston, MA, 1987, Translated from the German by Renate McLaughlin. MR 88i:30059b

[Gam69] T. W. Gamelin, Uniform algebras, Prentice-Hall Inc., Englewood Cliffs, N. J., 1969. MR 53 \#14137

[Gau98] P. M. Gauthier, Mittag-Leffler theorems on Riemann surfaces and Riemannian manifolds, Canad. J. Math. 50 (1998), no. 3, 547-562. MR 99h:30046

[Hav58] V. P. Havin, The separation of the singularities of analytic functions, Dokl. Akad. Nauk SSSR 121 (1958), 239-242. MR 20 \#4630

[Hav84] _ Golubev series and the analyticity on a continuum, Linear and complex analysis problem book (V. P. Havin, S. V. Hruščëv, and N. K. Nikol'skiǐ, eds.), Lecture Notes in Mathematics, vol. 1043, SpringerVerlag, Berlin, 1984, 199 research problems, pp. 670-673. MR 85k:46001

[Hav04] The separation of singularities of analytic functions with preservation of boundedness, Algebra i analiz 16, (2004), no. $1293-319$.

[HN01] V. P. Havin and A. H. Nersessian, Bounded separation of singularities of analytic functions, Entire functions in modern analysis (Tel-Aviv, 1997), Israel Math. Conf. Proc., vol. 15, Bar-Ilan Univ., Ramat Gan, 2001, pp. 149-171. MR 2002m:31018

[HO06] V. P. Havin and J. Ortega-Cerdà, A $\bar{\partial}$-problem related to separation of singularities of analytic functions.

[Hör90] L. Hörmander, An introduction to complex analysis in several variables, third ed., North-Holland, Amsterdam, 1990.

[HP84] G. M. Henkin and P. L. Polyakov, Prolongement des fonctions holomorphes bornées d'une sous-variété du polydisque, C. R. Acad. Sci. Paris Sér. I Math. 298 (1984), no. 10, 221-224. MR 85i:32021

[Jon83] P. W. Jones, $L^{\infty}$ estimates for the $\bar{\partial}$ problem in a half-plane, Acta Math. 150 (1983), no. 1-2, 137-152. MR 84g:35135

[MH71] B. S. Mitjagin and G. M. Henkin, Linear problems of complex analysis, Uspehi Mat. Nauk 26 (1971), no. 4 (160), 93-152. MR 44 \#4504

[Poi92] H. Poincaré, Sur les fonctions à espaces lacunaires, Amer. J. Math. 14 (1892), 201-221.

[Pol83] P. L. Polyakov, Continuation of bounded holomorphic functions from an analytic curve in general position into the polydisc, Funktsional. Anal. i Prilozhen. 17 (1983), no. 3, 87-88. MR 84k:32019 
[Sei04] K. Seip, Interpolation and Sampling in Spaces of Analytic Functions, University Lecture Series, AMS, Providence 2004. MR 05c:30038

[Val54] G. Valiron, Fonctions analytiques, Presses Universitaires de France, Paris, 1954. MR 15,861a

St. Petersburg State University. Department of Mathematics and Mechanics, 28 UniverSitetski PR., St. Petersburg, 198504, Russia

E-mail address: havin@VH1621.spb. edu

Burnside Hall, Department of Mathematics and Statistics McGill University, Montreal H3A 2K6, CANADA

Dept. Matemàtica Aplicada i Anàlisi, Universitat de Barcelona, Gran Via 585, 08071 BarCELONA, SPAIN

E-mail address: jortega@ub.edu 